\title{
L'écriture inventée : empirisme, constructivisme, socioconstructivisme
}

Invented spelling: empiricism, constructivism, socioconstructivism

Jacques Fijalkow, Jocelyne Cussac-Pomel et Dominique Hannouz

\section{(2) OpenEdition}

\section{Journals}

\section{Édition électronique}

URL : http://journals.openedition.org/educationdidactique/576

DOI : $10.4000 /$ educationdidactique. 576

ISBN : 978-2-7535-1622-9

ISSN : 2111-4838

\section{Éditeur}

Presses universitaires de Rennes

\section{Édition imprimée}

Date de publication : 1 octobre 2009

Pagination : 63-97

ISBN : 978-2-7535-1060-9

ISSN : 1956-3485

\section{Référence électronique}

Jacques Fijalkow, Jocelyne Cussac-Pomel et Dominique Hannouz, "L'écriture inventée : empirisme constructivisme, socioconstructivisme », Éducation et didactique [En ligne], 3-3 | Octobre 2009, mis en ligne le 01 octobre 2011, consulté le 08 décembre 2020. URL : http://journals.openedition.org/ educationdidactique/576; DOI : https://doi.org/10.4000/educationdidactique.576 


\title{
L'ÉCRITURE INVENTÉE : EMPIRISME, CONSTRUCTIVISME, SOCIOCONSTRUCTIVISME
}

\author{
Jacques Fijalkow, Jocelyne Cussac-Pomel \& Dominique Hannouz \\ Université Toulouse le Mirail
}

\begin{abstract}
Résumé : Demander à de jeunes enfants d'écrire un énoncé qui n'a pas fait l'objet d'un enseignement préalable, c'est les placer dans une situation où ils n'ont d'autre choix que de produire un graphisme à partir de ce qu'ils pensent être l'écriture et d'utiliser les connaissances dont ils disposent. Ce sont les productions réalisées dans cette situation que l'on appelle écriture inventée. On présente dans ce texte les recherches sur cette situation et relatives à l'acquisition portant sur des enfants francophones, en examinant les apports et les limites de la conception constructiviste, pour en venir enfin aux travaux réalisés dans une perspective socioconstructiviste.
\end{abstract}

Mots-clé : écriture inventée, constructivisme, Emilia Ferreiro, socioconstructivisme

Jacques Fijalkow

\section{Introduction}

Demander à de jeunes enfants d'écrire un énoncé qui n'a pas fait l'objet d'un enseignement préalable, c'est les placer dans une situation où ils n'ont d'autre choix que de produire un graphisme à partir de ce qu'ils pensent être l'écriture et à l'aide des connaissances dont ils disposent. Ce sont les productions réalisées dans cette situation que l'on appelle écriture inventée ${ }^{1}$. Elle peut être utilisée sur le plan pédagogique. Si ce n'est guère le cas actuellement en France, il en est différemment en Amérique du Nord où les débats sont vifs concernant la place à lui donner dans l'enseignement. Elle peut aussi être utilisée sur le plan de la recherche afin, paraphrasant Sigmund Freud, de connaître non pas les théories infantiles sur la sexualité mais sur l'écriture. Cette perspective est aussi celle des « connaissances naïves » (Lautrey, Rémi-Giraud, Sander et Tiberghien, 2009). C'est sur ce second plan que nous nous placerons ici, considérant que ce que l'on apprend en analysant l'écriture inventée n'est pas sans intérêt pour les acteurs du système éducatif.

\section{La domination empiriste}

Sur le plan épistémologique, la conception dominante dans le champ de l'acquisition de la lecture-écriture est l'empirisme, tant sur le plan de la recherche (sciences cognitives) que sur celui de la pratique et de l'opinion commune. Les orientations prises en France ces dernières années par le ministère de l'Éducation nationale, s'appuyant sur cette position épistémologique, sont venues réactualiser une conception que d'autres travaux - ceux que nous présenterons dans ce texte - abordent de façon très différente. Rappelons brièvement que, selon la conception empiriste, l'entrée dans l'écrit consiste essentiellement en l'acquisition par l'enfant de connaissances apportées de manière systématique par l'adulte, le rôle de l'enfant étant alors de les mémoriser puis de les mettre en œuvre (procéduralisation). La langue écrite étant considérée comme un codage de la langue parlée, son acquisition nécessite alors un enseignement qui privilégie le lien entre des unités d'oral supposées préexistantes chez l'enfant et des unités d'écrit à acquérir, le code. Ainsi, pour la conception empiriste, l'enseignement consiste pour l'essentiel à associer des unités d'oral à des unités d'écrit - les correspondances grapho-phonétiques (CGP) -, ce qui demande d'abord des activités de discrimination perceptive. Discrimination, mémorisation, procéduralisation constituent donc l'essentiel de l'apprentissage. Toutefois une brèche a récemment été ouverte dans cette conception - dont la simplicité, l'ancienneté et le fait qu'elle corresponde au sens commun expliquent la popularité - par le développement des recherches sur la conscience phonétique. Celles-ci ont en effet mis en évidence le fait que les unités d'oral, loin d'être déjà là quand commence l'enseignement, doivent au contraire faire l'objet d'un travail de la part de l'enfant. La découverte de la conscience phonétique, qui aurait pu déboucher sur une mise en question de la conception empiriste, a de fait conduit à systématiser un enseignement spécifique de conscience phonologique en mater- 
nelle et /ou au début du CP. Ajoutons enfin que cette conception ne s'intéresse pas à l'écriture inventée, car l'étude de celle-ci repose sur l'adoption d'une autre épistémologie - constructiviste ou socioconstructiviste. Nous nous en tiendrons dans ce texte aux seuls travaux réalisés selon cette autre épistémologie. Les travaux sur l'acquisition de l'écriture réalisés dans une perspective empiriste constituent un autre objet d'études qui demande une revue de question distincte. De façon semblable, Rieben (2003) distingue une " approche psycholinguistique génétique » et une « approche psycholinguistique cognitive »; nous nous en tiendrons ici à la première.

L'écriture inventée est un champ de recherches récent (Read, 1975, 1981; Chomsky, 1971). Read, linguiste, considérant des écrits produits par des enfants encore non ou peu scolarisés, a découvert que ces productions - loin d'être des suites de graphies plus ou moins aléatoires comme on le supposait jusqu'alors - présentent en réalité des liens avec l'oral de l'enfant. Les écrits de ces enfants, jusque-là considérés comme des simulacres sans grand intérêt, ont dès lors acquis un statut d'objet heuristique quant aux idées des enfants sur la langue écrite. Parallèlement, Carol Chomsky, psycholinguiste, a vu tout l'intérêt qu'un regard positif sur ce type d'écrits pouvait présenter et a incité les pédagogues à placer l'enfant en situation d'écriture afin de lui faciliter la tâche. En effet, si les enfants, avant tout enseignement formel, au lieu d'écrire n'importe quoi, sont déjà en mesure de produire des écrits réfléchis, il n'y a plus lieu de continuer à suivre la tradition suivant laquelle on enseigne d'abord à lire et plus tard à écrire, mieux vaut aller dans leur sens et les placer dès le départ dans des situations d'écriture. Grâce à ces travaux pionniers - qui pour le lecteur européen font écho à la place qu'avaient déjà proposé d'accorder à l'écriture des pédagogues tels que Montessori, Decroly ou Freinet - la voie était ouverte à la fois à des travaux de recherches sur la façon dont les enfants appréhendent la langue écrite et sur le parti que l'on peut en tirer sur le plan pédagogique. Dans ce texte, nous considérerons comme Read l'écriture inventée sous le seul angle de la recherche - l'écriture inventée comme moyen d'étudier l'enfant confronté à la langue écrite —, et exclurons donc les travaux d'intérêt pédagogique suivant la voie indiquée par Chomsky qui nécessiteraient une revue de question différente ${ }^{2}$.

\section{La rupture constructiviste}

La théorie d'Emilia Ferreiro.

Dans une conception constructiviste, l'acteur principal de l'entrée dans l'écrit est l'enfant et non pas l'adulte. En effet, l'entrée dans l'écrit est conçue comme une construction dont l'enfant est l'acteur et non pas le récepteur. Elle consiste donc moins en un apprentissage qu'en une suite de conceptualisations effectuées par l'enfant. Si divers chercheurs ont abordé ainsi l'étude de l'écriture inventée, le mérite principal en revient assurément à Emilia Ferreiro et à ses élèves (Ferreiro et Teberosky, 1979; Ferreiro et Gomez-Palacio, 1988, Ferreiro, 2000). Ferreiro, partant de la découverte de Read et Chomsky, mais la développant, propose d'aborder les écrits d'enfants dans un cadre théorique inspiré par la conception piagétienne de la connaissance : l'écriture est un objet de connaissance comme un autre (nombre, temps, espace...). L'acquisition de la langue écrite est une psychogenèse, un développement, dont il revient au chercheur d'identifier les étapes.

Nous nous centrerons dans ce texte sur cette façon d'aborder l'écriture inventée, en nous demandant si les positions affirmées dans ces travaux ne gagneraient pas à être revues à la lumière d'autres travaux et repensées dans un cadre plus large - socioconstructiviste.

La position de principe de Ferreiro se résume ainsi :

Tout semble indiquer que nous sommes en présence d'une réelle psychogenèse ayant sa propre logique interne, ce qui veut dire que les informations en provenance du milieu sont incorporées dans des systèmes interprétatifs dont la succession n'est pas aléatoire, bien que la durée de chaque moment d'organisation - et, par conséquent, des âges d'apparition - dépende d'un ensemble d'influences diverses (sociales, familiales, éducatives, individuelles, etc.). (Ferreiro, 2000, p. 59).

Ferreiro (1988) présente une vaste recherche longitudinale auprès de 1000 enfants hispanophones durant leur première année scolaire et analyse quelque 3680 productions d'écriture inventée. Avec chaque enfant, quatre entretiens individuels sont 
conduits durant l'année scolaire (début d'année, décembre, février, fin d'année). Diverses épreuves de lecture et d'écriture sont utilisées, avec chaque fois l'écriture inventée de mots et d'une phrase : l'enfant écrit sans modèle, à sa façon, puis justifie ses choix. On identifie alors les règles d'écriture de l'enfant. Une grande diversité de procédures apparaît, soit vingtdeux types d'écriture montrant que les conceptualisations de l'enfant sur l'écrit sont différentes de celles de l'adulte, qu'elles ne sont pas directement liées à ses compétences graphiques, et que le principe alphabétique se construit de façon progressive. Pour Ferreiro,

« [...] l'ordre de résolution des problèmes que l'enfant construit est semblable à une programmation idéale » (Ferreiro et Teberosky, 1979).

Cette programmation se développe en plusieurs temps successifs. Dans un premier temps, l'enfant fait tout d'abord des hypothèses de quantité et de variété de lettres, puis des hypothèses de correspondances oral/écrit : d'abord entre une lettre et une syllabe, puis entre une lettre et un phonème, enfin entre plusieurs lettres et un phonème. Voyons maintenant cette programmation plus en détail.

À son entrée dans l'écrit, l'enfant s'intéresse tout d'abord aux traits saillants de l'écriture (lettres, linéarité, direction), ce qui l'amène alors à la différencier du dessin. Un indice de la réalisation de ce saut conceptuel est que l'enfant marque désormais la différence entre le dessin et l'écriture en utilisant l'article indéfini pour le dessin, et le nom sans l'article pour l'écrit. Par exemple, pour l'image d'une chaise, il dira que c'est « une chaise " et, pour le texte sous l'image d'une chaise, que "c'est écrit chaise ". Plus précisément, l'enfant use de cette différenciation syntaxique pour signifier quand il parle de l'objet ou de l'image et quand il cherche à interpréter l'écrit, c'est-à-dire « ce qui est dit » dans les marques graphiques en rapport avec l'objet (Ferreiro, 1982, 2000).

À ce niveau, l'enfant pense que seuls les noms peuvent être écrits. Sa production est constituée de lettres et de pseudo-lettres sans correspondance aucune avec les sons. La lecture de sa production est globale, l'enfant faisant correspondre le tout sonore et le tout graphique. L'important pour lui est-ce qu'il veut écrire et non le résultat, c'est pourquoi ses premières productions sont fortement ressemblantes. Il n'en contrôle pas la quantité (pour un mot, certains enfants écrivent une ligne et d'autres une seule lettre) ou, quand il le fait, ce n'est pas pour répondre à un souci de différenciation. Peu à peu, il en vient à contrôler la quantité de lettres, sans toutefois la faire varier : les graphies sont alors semblables (les mêmes lettres dans le même ordre) ou différentes (une combinaison des mêmes lettres ou des lettres différentes).

Puis vient le moment où l'enfant cherche à quoi référer l'écriture. Il s'intéresse alors aux constituants de l'écrit que sont les lettres et à leur quantité, il imagine que le mot écrit est en lien direct avec le signifié et différencie dès lors la quantité de lettres en fonction des objets à écrire. Par exemple, il écrit moins de lettres pour caneton que pour canard (puisque le caneton est plus petit que le canard) ou la même suite de lettres répétées trois fois pour trois canards. Cette hypothèse de quantité l'amène également à penser que le mot doit avoir une quantité minimale de trois lettres (plus ou moins une) pour être lisible.

À un deuxième niveau, l'intention subjective ne suffit plus car l'enfant arrive à la conclusion que pour pouvoir lire des choses différentes, il doit y avoir une différence objective dans les écrits. Il s'applique alors à varier les lettres de ses écrits (variété intrafigurale). Il utilise également des lettres différentes pour chaque mot quand il possède un répertoire assez vaste, ou bien alterne l'ordre de celles-ci quand son répertoire est limité (variété interfigurale).

$\mathrm{Au}$ niveau suivant, l'enfant cherche une correspondance entre les aspects sonores et graphiques en accordant tout d'abord une valeur syllabique aux lettres. Ferreiro considère que ce changement est capital car l'enfant réalise une correspondance terme à terme stricte entre une unité orale (la syllabe) et une unité écrite (la lettre). Ceci lui permet progressivement de contrôler et d'anticiper la quantité de lettres à écrire. C'est pourquoi Ferreiro regroupe les deux premiers niveaux dans une période dite présyllabique et appelle syllabique ce troisième niveau. À ce niveau, l'enfant n'a pas besoin de connaître la valeur sonore des lettres, il segmente l'oral en syllabes et écrit généralement une lettre - n'importe laquelle - par syllabe. 
Le passage à la période suivante (syllabicoalphabétique) est marqué par l'apparition d'un conflit - quand l'enfant doit écrire un mot mono ou bisyllabique - entre l'hypothèse syllabique et le critère de quantité minimale précédemment élaboré. C'est ce conflit qui l'amène à faire une analyse de l'oral plus poussée, au niveau du phonème. Dans ses écrits des lettres représentent des syllabes et d'autres des phonèmes.

À la période suivante l'écriture est alphabétique : l'enfant écrit généralement une lettre pour chaque phonème ${ }^{3}$; enfin, à la dernière période, l'écriture est orthographique : l'enfant peut résoudre les problèmes d'orthographe et de segmentation de la phrase en mots parce qu'il est capable d'assimiler (au sens piagétien du terme) les exigences de la conventionalité orthographique de l'écrit.

Plus de vingt ans après ses premiers travaux, Ferreiro (2000) présente le développement de l'écriture dans un texte de synthèse qui décrit la façon dont l'enfant interprète les informations de son environnement. L'enfant y apparaît comme étant à la recherche active d'un système interprétatif général et cohérent d'écriture. Cette re-construction consiste à prélever des informations sur l'écrit et certaines d'entre elles entrent en conflit avec l'hypothèse provisoire que l'enfant fait à propos de ce système. Le conflit est résolu quand l'enfant a assimilé les informations dans le cadre d'une nouvelle hypothèse. Cependant, même si la dynamique du développement de l'écriture est très semblable d'un enfant à l'autre, chacun sélectionne les informations qu'il est capable d'assimiler et les exploite de façon différente.

Ferreiro, tout en réaffirmant l'existence d'une psychogenèse de l'écriture, prend en compte le contexte social en suivant le processus piagétien d'assimilation : c'est lors des interactions avec autrui que des conflits cognitifs apparaissent et que l'enfant construit des schèmes successifs en transformant les informations reçues. Notons que les étapes ne sont pas franchies au même moment en lecture et en écriture. Ainsi, l'hypothèse syllabique apparaît d'abord en lecture pour justifier une quantité de graphies produite selon une autre hypothèse. Par exemple, l'enfant écrit la suite graphique EOOAI pour « gato » («chat» en espagnol) en estimant la quantité de graphies possible et ce n'est qu'à la demande de relecture qu'il opère une segmentation syllabique en signalant successivement EO pour [ga] et OAI pour [to].

Dans ce dernier texte, une place est faite maintenant au contexte linguistique dans la psychogenèse puisque l'étape syllabique est décrite comme essentielle dans les « langues dont les frontières syllabiques sont clairement marquées " (espagnol, portugais, italien et catalan), alors que dans d'autres langues (anglais, français) les «premiers découpages peuvent ne pas être strictement syllabiques [...] l'enfant [cherchant] à découper le mot en des unités qui s'avèrent être plus larges que le phonème "(Ferreiro, 2000, p. 16). Le rôle de l'hypothèse syllabique comme moyen général de comprendre le lien entre le mot et les lettres n'est pas remis en cause par Ferreiro, mais apparaît plus ou moins marqué selon la langue.

\section{Les études d'ensemble ${ }^{4}$}

Les études présentées ici ont en commun le souci de prendre pour objet l'analyse des productions couvrant l'ensemble de la période au cours de laquelle il est possible de parler d'« écriture inventée ». Elles diffèrent cependant par leur orientation théorique. Les deux premières, travaillant en psychologie de l'enfant dans la foulée des travaux de Ferreiro, se proposent de vérifier dans quelle mesure l'évolution qu'elle décrit à partir d'un contexte sud-américain peut être retrouvée chez des enfants français. Les deux autres équipes, issues des sciences de l'éducation et des sciences du langage, se préoccupent également de l'écriture inventée dans sa totalité, mais, plus centrées sur l'objet "écriture inventée » que sur l'enfant et sa confrontation avec l'écrit, paraissent davantage soucieuses de voir comment les propriétés de la langue française se reflètent dans les écrits des enfants.

Les études de psycholinguistique génétique de l'université Lyon II

Partant des travaux de Ferreiro, Jean-Marie Besse dirige des travaux sur la façon dont les enfants français s'approprient les règles et le fonctionnement de l'écrit (Besse, 1989, 1990, 1992). Ces études, menées en étroite liaison avec des enseignants, ont 
aussi pour objectif de rendre ces derniers attentifs aux savoirs que l'enfant construit quand il écrit ce qu'il ne connaît pas, et d'élaborer conséquemment des conditions pédagogiques favorables à l'acquisition de l'écrit.

Besse observe l'évolution des conceptualisations de la langue française écrite dans le cadre d'une étude longitudinale, de la GS ${ }^{5}$ au CP. Les enfants participent à trois sessions d'écriture suivies d'un entretien. Le protocole, en plusieurs temps, comporte des tâches variées. À partir de la production écrite, de l'oralisation et du geste du doigt ${ }^{6}$, Besse établit cinq niveaux de conceptualisation qui sont proches de ceux évoqués par Ferreiro.

De manière générale, il constate que les enfants effectuent une activité cognitive spécifique :

- refus d'écrire autre chose que leur prénom ou leur nom de famille.

- production écrite sans conservation de l'oral : production d'écrits et non plus de dessins dont la signification n'est pas stable. Ils reviennent sur leur écrit en disant ce dont ils se souviennent. Aucun élément de la chaîne sonore n'est observable. La quantité de graphies est limitée. Certains n'établissent aucune différence entre mot et phrase, alors que d'autres emploient davantage de graphies pour les phrases que pour les mots.

- ajustement écrit / oral : essai de diverses modalités d'ajustement entre l'écrit et l'oralisation ou le geste du doigt. L'ajustement se produit au moment où l'enfant écrit ou a posteriori. C'est l'oral qui sert de guide pour l'écrit. L'écrit conserve une signification stable (les phrases légèrement transformées conservent leur sens). Selon de Gaulmyn (1988), " un passage essentiel est franchi lorsque l'enfant s'efforce pour dire ce qu'il y a d'écrit d'ajuster cette lecture à son écriture; il est conduit à corriger l'écriture pour la conformer à cette lecture».

- analyse phonétique: tentative d'effectuer cette analyse sur les mots ou les phrases. Ils produisent des valeurs sonores conventionnelles, particulièrement à l'initiale. Ils peuvent introduire des lettres non conventionnelles en étant conscients ${ }^{7}$ qu'un lecteur ne pourra pas les lire.
- hypothèses alphabétiques : intégration de lettres dont la valeur sonore est conventionnelle et sépare les mots de la phrase par des blancs graphiques.

Par ailleurs, Besse (1990) émet l'hypothèse que l'enfant met aussi en œuvre des compétences à partir d'un mot qu'il connaît bien : son prénom. L'étude consiste à demander à l'enfant d'écrire son prénom. Dans le cas où celui-ci est suffisamment lisible, l'entretien se poursuit; dans le cas contraire, l'adulte écrit le prénom de l'enfant. L'adulte cache alors une partie du prénom et laisse apparentes au moins trois lettres d'une syllabe; il conduit un entretien piagétien en demandant si la partie visible signifie encore le prénom de l'enfant et «ce que ça dit ». Les enfants (MS, GS, CP) participent à deux sessions d'écriture (mars, mai).

L'analyse montre aux deux passations que les enfants de MS ne différencient pas le tout des parties du prénom. La moitié des enfants de GS produisent une écriture correcte du prénom, mais ne différencient pas l'ensemble du prénom d'une partie de celuici. Pour Besse, la non-distinction entre la totalité du prénom et les parties constituantes apparaît au cours de la seconde période décrite par Ferreiro. De la même façon, en mars au CP, la moitié des enfants ne peut pas interpréter correctement une partie du prénom. Ces données indiquent que, même si l'enfant est capable de produire correctement son prénom, il ne procède pas ou peu à un travail cognitif lié à cette écriture ${ }^{8}$. La question se pose alors de la difficulté pour le jeune enfant à couper son prénom, pratique qui reviendrait à découper son identité ${ }^{9}$. Par ailleurs, sachant qu'il n'est pas possible d'établir un lien entre ces résultats et les pratiques enseignantes, car, de manière à pondérer l'effet maître les enfants ont été choisis dans des classes différentes du point de vue de leurs pratiques, les auteurs poursuivent l'analyse du rapport oral-écrit du prénom à partir d'un protocole différent.

La population est constituée de 27 enfants de GS interrogés en décembre-janvier et mai-juin, dont 14 sont revus en mars au CP. Dans cette situation l'enfant écrit son prénom; l'expérimentateur lui demande comment il a fait pour écrire; l'enfant écrit ensuite des phrases dans lesquelles son prénom figure au milieu de la phrase. Les résultats montrent que, dans le contexte d'une phrase, les enfants des niveaux production écrite sans conservation de l'oral et ajuste- 
Jacques Fijalkow \& al

ment écrit/oral peuvent ne pas écrire leur prénom ou encore ne pas écrire ce qui le précède. En revanche, quand le prénom est écrit, il peut servir d'indice pour accéder au niveau de conceptualisation supérieur : analyse phonétique. L'analyse des productions et de l'entretien conduit les auteurs à être vigilants sur l'interprétation que donne l'enfant a posteriori : celle-ci n'est pas toujours celle qui a guidé la production, mais peut découler de l'entretien.

Le prénom, même s'il constitue un objet singulier, semble donc suivre dans ses grandes lignes le processus de n'importe quel autre mot, si ce n'est que l'utilisation fréquente qu'en fait l'enfant peut lui servir de support pour progresser dans sa maîtrise de l'écrit en général.

Par la suite, Besse (1992) affine ses niveaux de conceptualisation, soit sept niveaux allant du moins élaboré vers le plus élaboré, mais qui ne sont pas à considérer comme une suite ordonnée d'étapes obligatoires.

1. L'écrit est une trace différente du dessin.

2. L'écrit est une trace qui se rapporte au référent (le nombre de graphies peut être en rapport avec les caractéristiques physiques du référent; par exemple moins de graphies pour écrire papillon que lion).

3. L'écrit est une trace relative à la durée de la chaîne sonore : coïncidence entre taille de l'écrit et durée de la chaîne sonore.

4. L'écrit renvoie à une analyse phonétique de l'oral : codage des unités d'oral.

5. L'écrit doit correspondre à l'ordre des éléments sonores.

6. L'écrit note plus que l'oral; l'enfant prend en compte les contraintes orthographiques.

7. L'écrit véhicule des conventions d'énonciation (types de textes, interlocuteurs...).

Cette description présente une vue d'ensemble des conceptualisations de l'écrit par l'enfant. De Gaulmyn (1988) note toutefois que «ce modèle de niveaux de conceptualisation de l'écrit est probablement trop simplifié. Les niveaux ne sont peut-être ni exclusifs l'un de l'autre, ni toujours successifs, mais les stratégies des enfants seraient combinables entre elles selon la situation [...] d'écriture ». De plus, le cheminement de chaque enfant est unique.

En définitive, partant de ces niveaux de conceptualisation, on peut regrouper les préoccupations graphiques des enfants en trois catégories (Besse, 2000), selon que l'enfant se préoccupe

- d'aspects figuratifs de l'écrit et non des aspects sonores de la langue (visuographie)

- d'établir des liens entre les unités d'oral et d'écrit (phonographie)

- de certains éléments orthographiques, les morphogrammes par exemple (orthographie)

De la même façon, Luis (1993) s'attache à comparer les étapes décrites par Ferreiro et celles qu'elle dégage de l'échantillon qu'elle étudie. La population, sélectionnée par les enseignantes de six écoles différentes, comporte pour chacune 3 garçons et 3 filles de GS de niveau hétérogène (en général, 2 bons, 2 moyens et 2 faibles). Les 36 enfants retenus en GS participent à une séance d'écriture en fin d'année scolaire et sont revus à trois reprises au $\mathrm{CP}$. Ils écrivent différentes phrases, dont une avec le prénom de l'enfant.

L'analyse longitudinale des productions permet de retrouver les niveaux décrits par Ferreiro. En revanche, Luis observe chez un enfant sur six une attitude de refus non observée dans l'échantillon de Ferreiro : les enfants ne produisent pas d'écrit en dehors de leur prénom et expliquent qu'ils n'ont pas appris à écrire ce qui est demandé. Les enfants se représentent l'écrit comme une transmission entre celui qui sait et celui qui ne sait pas, qui doit alors mémoriser la forme graphique. Selon elle, ce refus d'écrire révèle une conceptualisation idéographique : l'écrit n'est pas encore une représentation du signifiant sonore, mais une représentation conventionnelle du signifié.

Les études de psycholinguistique génétique de l'université de Toulouse-le Mirail

D’autres recherches sont réalisées en parallèle à l'université de Toulouse-le Mirail sous la direction de 
Jacques Fijalkow (Fijalkow et Fijalkow, 1991; Fijalkow et Liva, 1993; Fijalkow, Fijalkow, Pasa et Ragano, 2006). Celles-ci, tout en se réclamant d'une conception constructiviste, se situent cependant moins dans une perspective cognitive qui est celle de Ferreiro, que dans une perspective psycholinguistique, la préoccupation principale étant de voir comment l'enfant met en relation l'oral et l'écrit pour comprendre ce qu'est la langue écrite et se l'approprier.

La première étude (Fijalkow et Fijalkow, 1991) porte sur 153 enfants issus de 8 classes (3 GS, 3 CP, 2 CE1) engagées dans un projet de recherche-action, soit 269 protocoles au total (1 à 4 passations par enfant). Suivant la procédure de Ferreiro et GomezPalacio (1988), le matériel verbal comporte quatre mots et une phrase (rat, cheval, papillon, crocodile, Le rat mange du fromage). Appliquant une démarche de classement utilisée en psychologie génétique, ces protocoles sont alors répartis en groupes et sous-groupes, sans tenir compte du niveau scolaire de l'enfant, mais selon l'origine et de la grandeur du répertoire, et des modalités de construction des écrits. Ces groupes sont ensuite ordonnés selon un ordre de progression déterminé par les chercheurs. Les auteurs proposent ainsi les groupes suivants :

1. Idéosignes : les enfants se servent de signes figuratifs (dessins et tracés représentatifs de l'objet) et non figuratifs (boucles, ondulations, traits verticaux ou horizontaux, ronds, signes inventés par l'enfant). L'utilisation constante de signes créés par l'enfant et variant donc d'un enfant à l'autre, ainsi que l'absence totale de signes conventionnels tels que lettres et chiffres permet de parler d' « idéosignes »

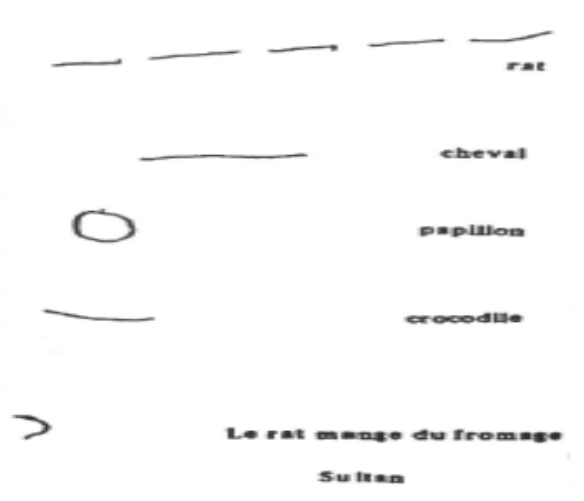

Figure 1 - Idéosignes
2. Pseudo-signes: les formes produites sont des pseudo-lettres (tracés semblables à une lettre mais qui ne se confondent pourtant pas avec une lettre) et des pseudo-mots (tracés continus qui, à la différence des tracés précédents, présentent une forte variabilité intra-mots; les unités qui les constituent, presque toujours non identifiables, sont des pseudolettres parmi lesquelles on croit parfois reconnaître une lettre). En bref, les formes produites imitent avec un certain bonheur les graphismes courants - lettres ou mots. La présence de ces « pseudo-signes » paraît caractéristique de ce groupe. Chaque enfant, utilise un petit nombre de signes différents, 3 ou 4 le plus souvent.

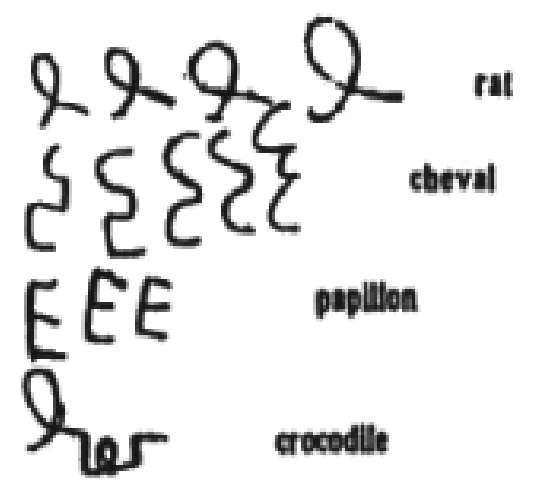

\section{Ctrisalian}

Figure 2 - Pseudo-signes

3. Mélange de signes en voie d'achèvement: on observe un mélange de signes conventionnels « en voie d'achèvement », c'est-à-dire de signes de différentes origines mais encore difficilement identifiables (lettres majuscules, lettres minuscules - parfois inversés, chiffres, pseudo-lettres). Chaque enfant dispose d'environ 10 signes différents.

4. Lettres isolées : on observe majoritairement des lettres - majuscules et/ou minuscules, parfois inversées - parmi lesquelles peuvent encore se trouver quelques pseudo-lettres. Plusieurs de ces lettres proviennent du prénom de l'enfant. Les enfants utilisent alors 4 ou 5 lettres.

5. Inflation littérale: seules des lettres sont utilisées, surtout majuscules; les lettres inversées et les pseudo-lettres sont rares. Les lettres disponibles sont très nombreuses et l'espace occupé par chacune 
d'elles enfle démesurément, c'est pourquoi ce groupe peut être défini en termes d'« inflation littérale ».

6. Jeu combinatoire de lettres: les signes utilisés sont essentiellement des lettres - majuscules ou minuscules, aisément identifiables. On ne trouve plus qu'exceptionnellement des lettres inversées, des pseudo-lettres et des chiffres. Ces lettres proviennent en grande partie du prénom de l'enfant. Le répertoire est de 7 à 9 lettres différentes. Pour obtenir des mots différenciés, les enfants utilisent trois procédures différentes : 1) Faire varier la composition des mots, 2) Faire varier la longueur de l'énoncé, 3) Écrire des mots qu'ils connaissent.

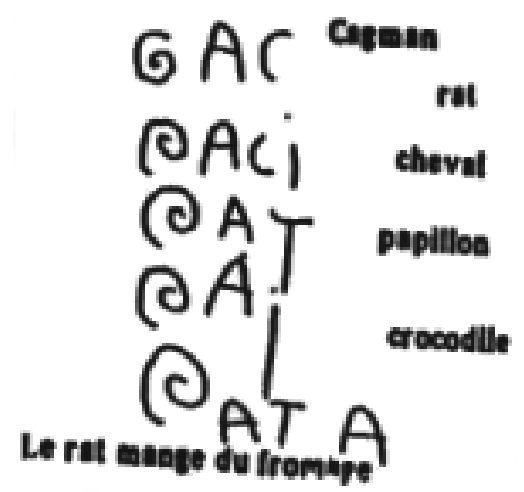

Figure 3 - Jeu combinatoire de lettres

7. Intervention de l'oral : un peu plus de la moitié des lettres proviennent du prénom de l'enfant. La phrase se présente comme une suite de segments. Si certains enfants continuent de procéder à un choix aléatoire des lettres, par tirages à l'intérieur de leur répertoire, d'autres commencent à prendre en considération l'existence de correspondances grapho-phonétiques et choisissent donc parfois une lettre correspondant plus ou moins (similitude phonétique) aux phonèmes qu'ils ont repérés à l'oral.

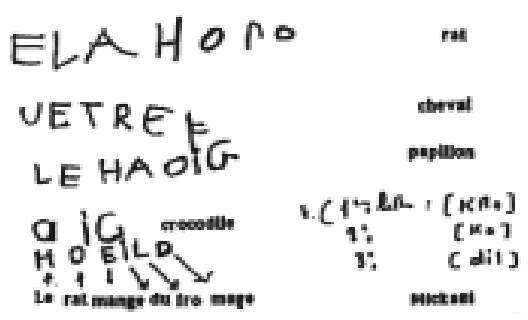

Figure 4 - Intervention de l'oral
8. Analyse phonétique partielle: La procédure dominante est de type phonétique. Les lettres qui apparaissent dans les productions écrites évoquent en effet certains phonèmes de l'unité à coder, qu'il s'agisse d'un mot donné isolément à l'enfant ou d'un mot qu'il a lui-même segmenté dans la phrase proposée. De manière générale, les mots écrits comportent des éléments qui, au moins partiellement, correspondent à leur structure phonétique : empiriquement, on peut, le plus souvent, à la seule vue de ce que l'enfant a produit, identifier parmi les mots possibles dans ce contexte quel est le mot que l'enfant a voulu écrire. La production écrite, exception faite des rares mots (déterminants) puisés directement dans le lexique interne, repose sur l'analyse de l'oral.

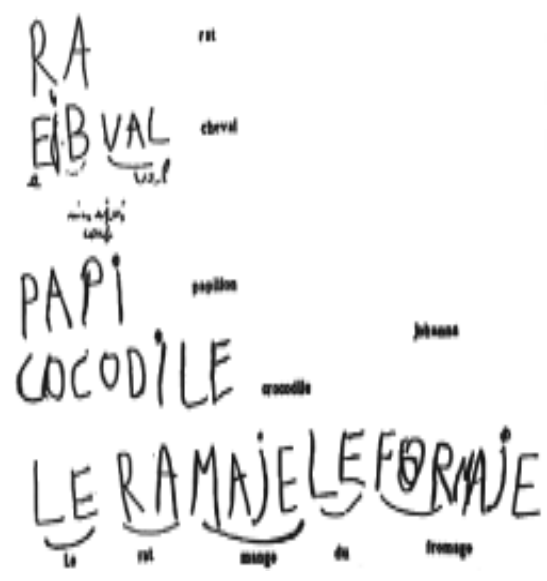

Figure 5 - Analyse phonétique partielle

9. Analyse phonétique complète: le répertoire de lettres est maintenant complet. L'analyse phonétique préside à la production écrite. Quelques exceptions concernent l'existence des mots puisés dans le lexique interne. Les productions ainsi réalisées sont désormais aisément identifiables par l'observateur adulte.

Ce premier essai d'analyse a une visée essentiellement exploratoire. Travail pionnier, à un moment où il n'existe pas encore de travaux en français sur l'écriture inventée, c'est une tentative globale de vérification, dans le contexte français, de l'existence des étapes indiquées par Ferreiro. Dans ce travail préliminaire, peu de place est accordée à la phrase et l'orthographe est ignorée. 
Une deuxième étude est réalisée peu après (Fijalkow et Liva, 1993) avec un objectif pragmatique : proposer aux acteurs du système éducatif une grille d'évolution de l'écriture inventée afin qu'ils puissent situer sur celle-ci le niveau d'un enfant de cycle $2^{10}$. Compte tenu de ce souci plus pédagogique, une place plus importante est faite à la phrase. L'étude porte sur l'ensemble des enfants de cycle 2 d'une ZEP ${ }^{11}$ (1 GS, 1 CP, 2 CE1) au cours d'une année scolaire et utilise les mêmes mots que précédemment, mais comporte deux phrases différentes (Le crocodile avale le papillon; Le rat monte sur le cheval). La grille se présente ainsi :

\begin{tabular}{|c|c|c|}
\hline Catégorie niv. 1 & Catégorie niv. 2 & Catégorie niv. 3 \\
\hline \multirow[t]{2}{*}{ Traitement figuratif } & L'enfant dessine & \\
\hline & L'enfant simule l'écriture & \\
\hline \multirow[t]{5}{*}{ Traitement visuel } & Pseudo-lettres + simulation & \\
\hline & Lettres et pseudo-lettres & \\
\hline & Lettres du prénom (majoritairement) & \\
\hline & Autres lettres (majoritairement) & \\
\hline & Graphie du mot réinvestie dans la phrase & \\
\hline Traitement de l'oral & $\begin{array}{l}\text { Phrase plus longue que le mot le plus } \\
\text { long }\end{array}$ & \\
\hline \multirow[t]{4}{*}{ Analyse des phrases } & $\begin{array}{l}\text { Phrase écrite avec une lettre pour chaque } \\
\text { mot }\end{array}$ & \\
\hline & Phrase segmentée en deux parties & \\
\hline & $\begin{array}{l}\text { Phrase segmentée en plus de deux } \\
\text { parties }\end{array}$ & \\
\hline & $\begin{array}{l}\text { Phrase segmentée en autant de parties que } \\
\text { de mots }\end{array}$ & \\
\hline \multirow[t]{7}{*}{ Analyse des mots } & $\begin{array}{l}\text { Mots écrits avec autant de lettres que de } \\
\text { syllabes }\end{array}$ & \\
\hline & $\begin{array}{l}\text { Mots écrits avec quelques correspondances } \\
\text { grapho-phonétiques (CGP) }\end{array}$ & Une lettre dans deux ou trois mots \\
\hline & & L'attaque des mots \\
\hline & & $\begin{array}{l}\text { Découpage en syllabes (au moins une } \\
\text { lettre par syllabe) }\end{array}$ \\
\hline & Écriture phonétique & $\begin{array}{l}\text { Trois ou quatre syllabes entières dans } \\
\text { l'ensemble de la production }\end{array}$ \\
\hline & & $\begin{array}{l}\text { Deux mots de plus de trois lettres écrits } \\
\text { phonétiquement }\end{array}$ \\
\hline & & Plus de deux mots de plus de trois lettres \\
\hline \multirow[t]{4}{*}{ Traitement orthographique } & Écriture orthographique partielle & Deux mots de plus de trois lettres \\
\hline & & $\begin{array}{l}\text { Plus de deux mots (mots isolés et/ou mots } \\
\text { d'une phrase) }\end{array}$ \\
\hline & Écriture orthographique systématique & Une phrase en écriture orthographique \\
\hline & & $\begin{array}{l}\text { Les deux phrases en écriture } \\
\text { orthographique }\end{array}$ \\
\hline
\end{tabular}

Tableau 1 - Grille d'évolution de l'écriture inventée 
Jacques Fijalkow \& al

L'analyse proposée repose sur la distinction de quatre modalités successives de traitement. Toutefois, la linéarité de l'évolution, le mélange de critères qualitatifs et quantitatifs, la séparation absolue entre mot et phrase seront jugés par la suite peu satisfaisants et demanderont donc une nouvelle élaboration.

Une autre tentative de systématisation a été présentée plus récemment (Fijalkow et al., 2006). Les données proviennent d'une recherche-action d'une durée de trois ans avec tous les enfants du cycle 2 d'une REP ${ }^{12}$ et d'une école de centre-ville. Les auteurs disposent de 10 passations au long des deux années où l'épreuve d'écriture inventée présente un intérêt (GS, $\mathrm{CP}$ ). Les résultats présentés portent sur 7 des enfants choisis au hasard, soit 70 passations au total. Les enfants ont à écrire 3 mots et 3 phrases (chat; Le chat boit du lait; souris; La souris mangeait le gâteau sucré; crocodile; Le crocodile dévora la méchante sorcière).

La démarche de recherche est différente des deux précédentes. En effet, le propos n'est plus maintenant ni de décrire la progression des enfants, ni de construire une grille d'évaluation, mais d'analyser maintenant l'écriture inventée à partir de deux principes théoriques qui ne présupposent plus une évolution universelle, linéaire et commune à tous les enfants, mais une évolution posée comme variable selon le contexte et selon l'enfant.

1) Selon le contexte: dans un contexte donné, les enfants disposent de divers mécanismes de réponse qui sont en nombre fini. En d'autres termes, le contexte dans lequel se trouvent les enfants offre un certain nombre de possibilités pour résoudre le problème. Les écrits des enfants n'ont donc pas une valeur universelle mais contextuelle, dépendant de l'offre contextuelle présente dans le milieu. Ainsi, dans le contexte particulier de cette étude, le contexte linguistique est celui de la langue française et le contexte didactique celui du langage entier; les conclusions obtenues ne permettent aucune généralisation à un autre contexte.

2) Selon l'enfant: chaque enfant a un mode d'acquisition singulier par rapport aux autres : il utilise les mécanismes disponibles dans le contexte dans lequel il se trouve, mais pas nécessairement tous et pas nécessairement dans le même ordre. Ce sont ces différences de choix et d'ordre qui sont analysées.
De ces principes découle une démarche de recherche en trois temps :

- identifier les mécanismes mis en jeu par au moins un enfant au moins une fois; en procédant à une analyse minutieuse et exhaustive des 70 protocoles recueillis, les auteurs produisent une liste d'environ 100 mécanismes de réponse;

- analyser à l'aide de cette liste toutes les réponses fournies par chacun des enfants;

- présenter une vue d'ensemble.

La question est alors de savoir comment les 7 enfants procèdent pour résoudre ce problème d'écriture dans ce contexte. L'auteur distingue quatre temps successifs, à l'intérieur desquelles apparaissent des réponses formellement différentes, mais qui sont considérées comme de niveau équivalent.

1. Premier temps

1.1. Les réponses semblent témoigner de peu d'intérêt pour les propriétés de l'écrit

1.1.1. Les formes utilisées ne sont pas des lettres

1.1.2. Le répertoire de lettres est limité

1.1.3. L'écrit ne permet pas de reconnaître une forme connue

1.1.4. L'enfant écrit une seule lettre pour chaque demande

1.2. Les réponses utilisent l'écrit de façon non conventionnelle

1.2.1. Les écrits sont répartis dans la page dans un ordre non conventionnel

1.2.2. Les écrits ont tous à peu près la même longueur

1.2.3. Les écrits sont de longueur croissante, afin de marquer les différences perçues à l'oral

1.2.4. Certains écrits correspondent à une ligne entière 
1.2.5. L'autocopie est utilisée massivement sur le plan vertical

1.2.6. L'autocopie est utilisée massivement sur les plans vertical et horizontal

1.3. Les réponses sont centrées sur les propriétés formelles de l'écrit (ordre de haut en bas, nombre minimum et maximum de lettres, variété intra et interfigurale, différence de longueur mot < phrase, un énoncé par ligne, mot réécrit dans la phrase, écriture des articles)

1.4. Les réponses sont centrées sur les grandes unités de l'oral

1.4.1. Écriture par couples mot / phrase (l'enfant isole des blocs composés d'un mot et de la phrase qui contient ce mot)

1.4.2. Différenciation entre le mot et la phrase (l'enfant écrit tous les mots suivant un modèle particulier, et toutes les phrases suivant un autre modèle)

\section{Second temps}

La perception de l'oral s'affine. L'enfant identifie des unités d'oral plus courtes : non plus seulement mot ou syllabe, mais aussi phonème. On peut distinguer alors 4 procédés plus ou moins contemporains :

Procédé 1: phonémisation (chat, souris, crocodile)

Procédé 2 : remplissage (lettres sans correspondance phonétique avec la demande)

Procédé 3 : écriture lexicale (mots courts et fréquents : articles...)

Procédé 4 : écriture littérale : l'enfant écrit une lettre dont le nom correspond à une syllabe du mot à écrire

\section{Troisième temps}

Tout ce qui est écriture inventée disparaît au bénéfice d'une écriture contrôlée :
3.1. Peu de remplissage mais écrits massivement phonographiques

3.2. Confusions entre unités voisines : erreurs de type phonémique

\subsection{Acquisition d'unités lexicales précises}

3.4. Acquisition de morphogrammes lexicaux

4. Quatrième temps

Les écrits sont normés, sauf quelques des difficultés qui persistent

4.1. Certains phonèmes confondus avec des graphèmes homophones ou des phonèmes voisins

4.2. Morphogrammes grammaticaux erronés ou absents

4.3. Erreurs lors des séquences consonne-consonne

4.4. Erreurs sur les mots longs et / ou comportant un phonème apparaissant à plusieurs reprises à peu de temps d'intervalle

4.5. Écriture des semi-consonnes (ex. boit, sorcière)

Les études fondées sur des caractéristiques linguistiques.

À la différence des psychologues de l'enfant s'efforçant de voir dans quelle mesure il est possible de retrouver les étapes décrites par Ferreiro, les études suivantes, affranchies de ce souci, prennent davantage en compte la complexité de la langue telle qu'elle résulte des études de linguistique et, se fondant sur celles-ci, s'intéressent à inventorier la multiplicité des réponses des enfants, sans souci de les hiérarchiser dans le temps. Plusieurs auteurs ont remarqué le fait que l'on peut observer à un moment donné chez l'enfant des stratégies qu'un modèle de développement par étapes (celui de Ferreiro ou d'autres) présente comme successives (De Gaulmyn, 1988; Rieben et al., 2005; Saada-Robert, 2007), mais nous ne présenterons ici que ceux qui présentent ce point de manière détaillée. 
Jacques Fijalkow \& al

Les études québécoises.

Morin et Montésinos-Gelet étudient l'entrée dans l'écrit d'enfants francophones au Québec (5.57 ans), en maternelle et première année : écriture inventée de mots et commentaires post-graphiques des enfants. Partant de la représentation du français écrit comme plurisystème (Catach, 1995), elles invitent à nuancer le modèle linéaire du développement de l'écriture en 3 stades (logographique - alphabétique - orthographique) pour privilégier un modèle multidimensionnel plus souple, davantage apte à rendre compte du fait que, dès le début de l'apprentissage, l'enfant recourt de façon concomitante à des stratégies variées (lexicale, analogique, phonologique, syllabique). En effet, bien que l'enfant acquière rapidement une bonne maîtrise de la dimension phonogrammique (dès l'année de maternelle), il n'est pas uniquement centré sur cet aspect de l'écrit. Il introduit des morphogrammes dans ses écritures ( $4,95 \%$ des enfants de fin de GS), et ce même s'il n'a pas achevé la construction liée aux aspects phonologiques. Les commentaires métagraphiques témoignent d'une préoccupation précoce de la dimension morphogrammique de la langue (Montésinos-Gelet et Morin, 2001; Morin, 2004a ; Morin, 2004c; Morin et Montésinos-Gelet, 2004). La dimension morphographique du français est ainsi lentement intériorisée par l'enfant, d'abord dans l'activité de compréhension, moins coûteuse en termes de contrôle cognitif que dans l'activité de production (Morin et MontésinosGelet, 2005). Morin l'observe en particulier pour la construction des marques morphographiques singulier/pluriel (Morin, 2004b).

Par ailleurs, l'analyse spécifique des commentaires métagraphiques de l'enfant montre qu'il n'est pas forcément conscient des stratégies qu'il utilise. Morin observe qu'en phase de construction de la dimension phonographique, il use d'un langage verbal en cours d'écriture (destiné à réguler la tâche) avant d'être capable de produire a posteriori des commentaires métalinguistiques, signes d'une prise de conscience de la relation entre les sons et les signes écrits. Quand il s'intéresse à la dimension orthographique, le langage verbal en cours d'écriture se manifeste moins et les commentaires métalinguistiques post-production sont plus fréquents. Les commentaires métacognitifs, témoins de la conscience des stratégies, sont encore rares (Morin, 2005).
Ces études confirment donc l'intérêt qu'il y a à prendre en considération les caractéristiques linguistiques du français. Au contraire d'autres langues plus transparentes, la connaissance des CGP ${ }^{13}$ donne peu accès aux graphèmes orthographiques. La langue française, parce qu'elle est une langue orthographique opaque, impliquerait une double fondation : phonographique et morphographique.

Les études linguistiques de l'équipe CNRS-HESO.

Dans une perspective également basée sur des caractéristiques précises de la langue écrite, Jaffré (1992) estime que l'acquisition de l'écrit est propre à chaque enfant. Pour lui, certaines procédures « doivent composer des sous-systèmes communs à plusieurs individus sinon à l'ensemble ». Ces soussystèmes constitueraient un "modèle de l'acquisition souple et modulaire $»(J a f f r e ́, 1991)$.

Prenant comme base théorique les travaux de Hjelmslev (1971), Jaffré interprète les procédures graphiques des jeunes enfants au regard des systèmes linguistiques tels que Hjelmslev les catégorise. Dans ce cadre, dans les systèmes d'écriture coexistent les unités dépourvues de sens, les cénèmes (phonèmes, syllabes) et celles qui en sont pourvues, les plérèmes (morphèmes, mots). Les enfants doivent donc "construire des formes visibles de la signification (plérèmes) et les formes visibles de l'oralité (cénèmes) ».

À partir de productions relevées dans des ateliers d'écriture auxquels des groupes de 8 à 10 enfants de 5-6 ans participent une fois par semaine, l'auteur observe des éléments graphiques représentatifs d'opérations psycholinguistiques. Les enfants écrivent en fonction des projets élaborés en classe (recettes, récits, visites...). L'auteur propose une hiérarchisation des procédures.

Les procédures non fonctionnelles sont distinguées de celles qui sont fonctionnelles. Dans la première catégorie, figurent les premières lettres écrites par l'enfant qui ne remplissent aucune fonction linguistique. L'enfant peut dire : «J'ai dessiné des lettres ».

Au sein des procédures fonctionnelles, la distinction est faite entre les productions pseudo-graphiques et graphiques. 
Dans les productions pseudo-graphiques l'enfant aligne des lettres et leur attribue une signification. Ces procédures plérémiques sont les prémices de l'entrée dans l'écrit puisque l'enfant représente du sens.

L'auteur catégorise les procédures graphiques selon des niveaux différents :

- logographiques

- phonographiques

- épellatives

- syllabiques

- logo-syllabiques

- phonogrammiques

- digrammiques

- logo-phonogrammiques

- distributionnelles

Les procédures logographiques.

Des séquences de lettres, aléatoires ou non, apparaissent dans les productions. Le prénom et d'autres mots familiers servent de source graphique.

Les procédures phonographiques.

L'entrée dans l'écriture cénémique se produit à partir du moment où l'enfant tente de noter des unités linguistiques non significatives.

L'enfant cherche à noter certains aspects sonores de la langue. Il peut utiliser le nom des lettres (procédure épellative), même s'il n'en connaît pas la transcription. En revanche, quand il connaît le nom et la transcription d'une lettre, l'enfant fait des choix plus précis. L'auteur observe que les voyelles $\mathrm{A}, \mathrm{I}, \mathrm{O}$ et $\mathrm{U}$ sont plus fréquemment employées que les consonnes. La connaissance du nom de certaines consonnes (B, C, D, R, S) permet aux enfants de coder des syllabes (ex. T pour [te] dans pâté) ou des segments infra-syllabiques (ex. R pour [ $\bigotimes$ r] dans faire, vert).
Les enfants peuvent repérer à l'oral le nombre de syllabes et les représenter par autant de lettres (procédure syllabique), même si celles-ci n'ont aucune relation sonore avec les syllabes à coder (ex. Une fois, une petite $=$ AAAAA). En revanche, dans certains cas, l'association entre une lettre et une syllabe n'est pas systématique. Par exemple, quand les enfants connaissent les digrammes, l'auteur observe deux lettres pour une syllabe (ex. dans = AN). Par ailleurs, dans certaines productions, le codage des syllabes de structure complexe (CVC, CCV, CVCC...) laisse aussi apparaître plus d'une lettre pour une syllabe (ex. spectacle $=$ CPTCL $)$.

L'emploi d'une lettre par syllabe produit des segments graphiques courts dans le cas des mots mono ou bisyllabique (ex. parfum = AF). L'ajout de lettres non fonctionnelles apparaît comme une solution pour conférer alors au mot une taille minimum (ex. parfum = AFOIP).

Les procédures phonogrammiques.

Quand chaque phonème du mot est associé à une lettre et réciproquement, la production est qualifiée de phonogrammique. Les lettres employées peuvent être ou non conventionnelles (ex. voulons = VÉLÉ). Les écarts à la norme peuvent résulter de confusions idiolectales (ex. [o] / [ø]; rôti $=$ RETI), de confusions sourde/sonore $($ ex. bifteck $=$ PIFK), de confusions de lettres (ex. café = GAFÉ; phonème prononcé $[\mathrm{k}]$ ), d'adjonction par surarticulation (ex. voir = VOUOIR) ou d'omission de phonèmes dans les groupes consonantiques (ex. blanc $=\mathrm{LAN})$.

Les procédures digrammiques.

L'auteur observe l'emploi des digrammes dans les procédures syllabiques et a fortiori dans les procédures phonogrammiques. L'emploi des digrammes varie selon qu'il existe ou non des formes graphiques concurrentes phonologiques. Par exemple, le graphème OU qui code le phonème $[u]$ est la seule transcription possible et apparaît plus précocement que la forme AU qui est un des codages du phonème [o].

Les procédures logo-phonogrammiques.

Ces procédures logo-phonogrammiques renvoient à des productions exhaustives de phonogrammes. 
Jacques Fijalkow \& al

Les mots sont alors des squelettes phonogrammiques (ex. pays = PÉI).

Les procédures distributionnelles.

Les phonogrammes sont employés le plus souvent sans tenir compte de la compétence distributionnelle. Ainsi, le graphème $C$ peut figurer à côté d'un A pour coder [sa] (ex. laissa = LÉCA). L'emploi des graphèmes respectant le principe distributionnel semble relever du hasard (ex. assis $=\mathrm{ACI}$ ).

Les travaux de David (2002, 2003a, 2003b), comme ceux de Jaffré, s'inscrivent dans le paradigme de recherche qu'ils dénomment « Linguistique génétique de l'écrit ».

"Il s'agit d'un paradigme de recherche qui associe l'étude de la morphogenèse de l'écriture dans l'humanité (perspective historique), de l'évolution des systèmes d'écriture actuellement en usage (perspective comparatiste) et de l'ontogenèse de l'écrit chez l'homme, l'enfant et l'adolescent (perspective psycholinguistique). »(David, 2002, p. 1)

Dans le dernier des trois cas indiqués, une étude sur un cas isolé s'intéresse à la phonographie et à la morphographie (2003a), aspects auxquels s'ajoute la sémiographie quand l'étude porte sur un groupe d'enfants (2002) ou encore les premières constructions orthographiques (2003b).

Les recherches réalisées pour décrire l'évolution générale de l'écriture inventée en français, tant du fait des différences dans le temps au sein d'une même équipe que d'une équipe à l'autre, échouent à retrouver l'évolution décrite par Ferreiro dans le cas du français. Même si de-ci de-là les auteurs repèrent quelques particularités de l'écriture enfantine énoncées par Ferreiro, le tableau général de l'évolution qu'ils présentent est clairement différent du sien. Pour expliquer ce fait, sans doute faut-il faire une place aux difficultés méthodologiques que rencontre la mise en ouvre dans les sciences humaines et sociales du principe de réplication repris des sciences de la nature. Il est vraisemblable toutefois que ces difficultés méthodologiques ne sauraient suffire à rendre compte de la différence entre l'évolution indiquée par Ferreiro et les présentations effectuées par les auteurs cités. Renonçant donc à examiner dans son ensemble la psychogenèse que propose Ferreiro, et puisque néanmoins certaines des caractéristiques qu'elle énonce ont pu être repérées, des recherches ont été entreprises centrées sur quelques concepts clés des périodes indiquées par Ferreiro plutôt que sur un schéma évolutif qui, dans son ensemble, se dérobe à l'observation.

\section{Les études sur des périodes précises}

Le fait que les études d'ensemble aient échoué à confirmer clairement l'évolution d'ensemble présenté par Ferreiro, ne signifie pas que la description spécifique faite de telle ou telle étape ne se révèle acceptable. C'est ce que les travaux suivants, effectués dans le contexte de psychologie de l'enfant indiqué plus haut, se proposent d'examiner.

De façon synthétique, Ferreiro présente ainsi les systèmes interprétatifs successifs de l'enfant et les niveaux de conceptualisation qui y correspondent :

2. La première période est caractérisée par la recherche de paramètres distinctifs entre les marques graphiques figuratives et les marques graphiques non figuratives, ainsi que par la constitution de séries de lettres en tant qu'objets substituts et la recherche des conditions d'interprétation de ces objets substituts.

3. La deuxième période est caractérisée par la construction de modes de différenciation entre les enchaînements de lettres, en jouant alternativement sur les axes de différenciation qualitatifs et quantitatifs.

4. La troisième période est celle qui correspond à la phonétisation de l'écriture, qui culmine dans la période alphabétique. (Ferreiro, 2000, p. 16)

La question est alors de savoir si les trois périodes indiquées par Ferreiro et les propriétés qui les caractérisent peuvent être retrouvées dans un contexte francophone.

La première période

Des travaux récents effectués en France portent précisément sur cette première période et sur la confusion initiale entre dessiner et écrire chez les 
tout jeunes enfants (Noyer, 2005; Noyer-Martin et Baldy, 2005, 2008). Dans ces investigations (transversales, longitudinales, et à longitudinales transverses) les enfants sont invités tour à tour à dessiner un objet et à écrire son nom. L'analyse fait apparaître quatre stratégies d'écriture : 1) « Picturale » : tracés identiques pour le dessin et l'écriture, ou différents mais liés spatialement; 2) «Spatiale »: l'écriture discrète (petits traits horizontaux) ou continue (tracé linéaire); 3) «Sémiotique »(lettres inventées ou en miroir, mélangées à des chiffres ou à des lettres, ou lettres exactes) ; "Phonographiqu e »(syllabique ou syllabico-alphabétique ou alphabétique); «Orthographique » (mot correct, sans omission ni ajout). Ces stratégies corroborent les résultats obtenus antérieurement dans les études de psychologie génétique de Besse (1989, 1990, 1992) et de Fijalkow et Fijalkow (1991) et Fijalkow et Liva (1993). Toutefois, à ne considérer que la première période, est-on assuré que tous les enfants aient impérativement à passer par un temps au cours duquel le dessin et l'écriture devraient être différenciés l'un de l'autre ou s'agit-il d'un phénomène qui, si banal soitil, ne vaut que dans un contexte culturel donné? Il se pourrait en effet que, dans des conditions sociales particulières, les enfants accèdent directement aux signes conventionnels que constituent les lettres. Plusieurs faits invitent à se poser la question.

Le premier, d'intérêt ethnographique, concerne plus précisément le contexte religieux. En effet, des enseignants de Mayotte - collectivité départementale d'outre-mer française_située dans l'archipel des Comores au nord-ouest de Madagascar et dont $90 \%$ de la population est musulmane - ont certifié à un des auteurs de ce texte que les enfants placés en situation d'écriture inventée ne répondent jamais par un dessin à une demande d'écriture de l'adulte. Il faut savoir en effet que l'islam interdit la représentation du corps humain. Il se pourrait donc selon eux que, compte tenu de cet interdit, le dessin ne soit pas utilisé comme moyen de représentation par ces enfants comme il l'est dans le contexte dans lequel ont lieu la plupart des études. On peut d'ailleurs penser que le même résultat serait observé dans un contexte juif traditionnel, du fait de l'existence de ce même interdit de reproduction de la figure humaine. Force est donc de supposer que les enfants élevés dans ce type de contexte aborderaient directement l'apprentissage des lettres, objets d'ailleurs hautement valorisé dans les deux contextes religieux évoqués. Dans ce cas, l'existence de cet interdit éviterait donc à ces enfants les difficultés que rencontrent ceux qui grandissent dans un contexte où la distinction entre dessin et écriture peut leur poser les problèmes qu'analyse fort judicieusement Ferreiro et qui en font pour elle le premier temps de la conceptualisation de l'écriture. Il se pourrait donc que ces problèmes résultent moins d'une nécessité intrinsèque au développement que du fait que celui-ci se déroule dans un contexte qui accepte dans un premier temps un mode de représentation figuratif qui, dans un second temps, entre en conflit avec le mode conventionnel proposé par la suite. Ces remarques qui ne reposent que sur des informations indirectes ne sont évidemment qu'une hypothèse et ne sauraient être retenues que sous la réserve des indispensables validations empiriques à effectuer. Elles permettent toutefois de focaliser l'intérêt des chercheurs sur un aspect du comportement des enfants - dessiner en réponse à une demande d'écriture - qui jusque-là relevait de l'évidence, et d'ouvrir aux recherches ci-dessous.

À côté de cette hypothèse ethnographique, à valeur uniquement heuristique, l'observation a été faite dans maintes classes d'école maternelle que, dans les classes où l'écrit est très présent dès la petite section (livres de jeunesse, abécédaires, ordinateur...), on ne voit pratiquement jamais d'enfants écrire autre chose que des lettres quand on les place en situation d'écriture inventée. L'absence de dessins dans les productions des enfants scolarisés dans un contexte riche en écrits semble alors imputable à un facteur empirique qui résulte de la fréquentation assidue des écrits, caractéristique de cette pédagogie. Ainsi, dans une étude en cours, Nathalie Prévost n'observe aucun dessin dans les productions écrites des enfants. Son étude porte sur 157 enfants de six écoles maternelles au Québec ${ }^{14}$. Le protocole comporte notamment l'écriture de sept mots (ami, girafe, idée, cerise, escargot, hibou, macaroni) effectuée deux fois au cours de la maternelle (octobre et mai), soit 2198 productions écrites au total. On peut donc penser que, dans ce type de classes, l'omniprésence de l'écrit conduise l'enfant à concevoir très tôt et très clairement qu'un énoncé oral s'écrit avec des lettres et non au moyen du dessin. Ceci invite à supposer que, tout comme l'interdit est susceptible d'empêcher l'apparition de dessins dans le premier cas, ici un environnement propice à la connaissance des lettres 
Jacques Fijalkow \& al

incite les enfants à les utiliser très tôt, sans conflit avec un système antérieur de représentation.

S'il ne nous a pas été possible de conduire une observation objective dans les deux contextes religieux évoqués, et pour aller néanmoins au-delà de ce qu'apporte l'observation dans les classes, il est apparu intéressant de s'appuyer sur un essai de vérification expérimentale. Celle-ci consiste en une intervention de didactique expérimentale qui, de manière certes limitée en durée par rapport à ce que peuvent vivre les enfants dans les classes évoquées, tente néanmoins de voir si un apport d'écrit massif auprès de jeunes enfants peut effectivement avoir pour effet de les inciter à produire des lettres plutôt que de dessiner (Sarris, 1996).

Le protocole expérimental est donc une reproduction en laboratoire des situations que peuvent rencontrer les enfants à l'école : un groupe expérimental 1 dans lequel l'écrit est très présent, un groupe expérimental 2 où il est moins présent, et un groupe témoin sans intervention ajoutée. On s'attend à ce que, selon le groupe, la production de dessins diminue et qu'augmente la proximité avec les normes d'écriture. Ainsi, la variable indépendante est l'entraînement à l'utilisation de l'écrit (fort / faible) et la variable dépendante est l'emploi de lettres de l'alphabet. Dans cette intervention, la tâche d'écriture inventée consiste à demander aux enfants d'écrire tour à tour des mots isolés (rat, cheval, papillon, crocodile) et une phrase qui reprend un des mots isolés (Le rat mange du fromage). Cette tâche sert de prétest et de post-test. La population se compose de 30 enfants de MS (4 ans en moyenne). Ils ont été retenus à l'issue d'un prétest d'écriture inventée du fait que, en réponse à la demande d'écriture, tous dessinaient et n'écrivaient pas de lettres. Ils ont ensuite été répartis en trois groupes de 10 enfants que l'on a appariés à partir de l'âge, du sexe et du milieu social. L'intervention didactique qui a lieu ensuite comporte 30 séances, différentes selon le groupe. Dans le groupe expérimental 1 , la séance comporte trois temps : 1) l'adulte lit une histoire à l'enfant en suivant le texte du doigt; 2) l'enfant dicte un mot de l'histoire à l'adulte et celui-ci l'écrit sous ses yeux en invitant l'enfant à l'observer; 3) l'enfant copie le mot qui a été écrit par l'adulte. Dans ce groupe, la familiarisation avec la langue écrite porte donc à la fois sur la lecture et sur l'écriture ${ }^{15}$. Dans le groupe expérimental 2, la séquence comporte deux temps : 1) l'adulte lit une histoire à l'enfant en suivant le texte du doigt ; 2) l'enfant fait un dessin correspondant à l'histoire qu'on vient de lui lire. Dans ce cas, la séquence - lecture faite à l'enfant, puis dessin par celui-ci - simule davantage un type de pratique assez courant dans les classes. Soulignons que si, dans le groupe expérimental 1, lecture et écriture interviennent l'une et l'autre, dans le groupe expérimental 2, l'écriture est remplacée par le dessin. Les enfants du troisième groupe (témoin) demeurent dans leur classe et ne passent donc que le prétest et le posttest. L'analyse des productions écrites par les enfants, avant et après l'intervention didactique, est effectuée à l'aide d'une grille ${ }^{16}$ en huit points (du dessin à l'écriture conventionnelle ${ }^{17}$, en passant par des tracés simulant l'écriture, la production de pseudo-lettres, etc.). La comparaison des réponses entre prétest et post-test, tout comme entre les trois groupes au posttest, fait apparaître la hiérarchie attendue : le groupe 1 utilise plus l'écriture conventionnelle que le groupe 2 , et celui-ci plus que le groupe témoin.

Partant de là, on peut se demander si, dans le cas où les enfants répondent par des dessins plutôt que par des lettres à la demande d'écriture, ce n'est pas du fait de leur ignorance plutôt que d'une nécessité liée à leur développement.

La seconde période

La seconde période que distingue Ferreiro appelle à son tour plusieurs commentaires. Selon elle, pour l'enfant, lors de cette seconde période,

Ce qui est interprétable n'est pas une lettre isolée mais une série qui doit remplir deux conditions formelles essentielles : avoir une quantité minimale et ne pas présenter la même lettre répétée (variété intra-figurale) (Ferreiro, 2000, p. 21)

La première condition pour qu'un écrit soit considéré comme tel par l'enfant serait donc qu'il comporte un minimum de lettres (quantité minimale). Cette proposition a donné lieu à la construction d'un dispositif destiné expressément à examiner cette proposition (Diéguez et Durand, 2006). La tâche proposée aux enfants consiste à leur demander de dire, pour les écrits qui leur sont présentés un à un sur l'écran de l'ordinateur, si «cette écriture peut 
être lue par une grande personne ou pas». Ce jugement de lisibilité est effectué sur des pseudo-mots soigneusement construits de telle sorte qu'ils soient de longueur variable tout en respectant les propriétés du français écrit. 27 pseudo-mots sont présentés un à un aux enfants : 18 items de 1 à 18 lettres, un item occupant toute la ligne, et 8 items doublant des items de longueur diverse (afin d'examiner la constance des réponses). L'épreuve a été passée par 55 enfants de GS. Les résultats confirment clairement qu'il existe bien une longueur minimale pour qu'un mot soit considéré comme lisible. Plus précisément, pour la quasi totalité des enfants, tout écrit à partir de cinq lettres est acceptable. En deçà de 5 lettres par contre les acceptations sont moins nombreuses et d'autant moins nombreuses que la longueur diminue. Il y a donc là une confirmation nette de la condition de longueur minimale formulée par Ferreiro.

La question de la variété intrafigurale a donné lieu à une étude comparative entre des enfants de langue maternelle italienne ou espagnole (Ferreiro, Pontecorvo et Zucchermaglio, 1987). Cette comparaison part du fait qu'en espagnol les lettres redoublées sont rares, alors que c'est l'inverse en italien. La variété intrafigurale est donc plus forte en espagnol qu'en italien. On peut dès lors se demander si cette différence a un effet chez des enfants d'école maternelle et de première année, c'est-à-dire si les enfants espagnols et italiens prennent en compte différemment cette propriété de l'écrit. L'étude consiste à leur présenter des cartes sur lesquelles figure un mot. Les mots, présentés deux à deux, sont les mêmes, avec cette différence que l'un comporte une lettre redoublée et l'autre non (ex. piza / pizza). L'expérimentateur s'entretient avec les enfants sur les couples qui leur sont présentés. Les auteures distinguent cinq groupes de réponses. Il apparaît alors que, dans les deux contextes, un groupe (groupe III) conteste la présence d'une lettre redoublée, ce qui indique une exigence de variété intrafigurale et témoigne donc d'un intérêt pour cette particularité linguistique. Toutefois, alors qu'on aurait pu s'attendre à ce que cette mise en cause apparaisse plus souvent chez les hispanophones, c'est l'inverse qui se produit : les enfants italiens sont plus nombreux (39,4\%) que les enfants hispanophones (29\%) à faire ce type de commentaire. Si l'étude valide donc l'hypothèse d'une exigence de variété, il ne semble pas que celle-ci soit le reflet pur et simple des propriétés de la langue utilisée, comme le supposerait la conception empiriste, car dans ce cas les enfants espagnols auraient été les plus nombreux à contester la présence de doubles lettres. Ces résultats semblent donc indiquer que les réponses des enfants viennent de leur réflexion sur la langue plutôt que des propriétés spécifiques de celle-ci.

Afin d'évaluer l'hypothèse intrafigurale en français, une autre recherche a été réalisée (Masse et Tenes, 2006). Le dispositif construit à cet effet consiste à présenter aux enfants des pseudo-mots un à un sur ordinateur et à leur demander un jugement d'acceptabilité ("Ce qui est écrit, ça peut se lire?», "Ça existe?», "Est-ce que tu penses que la machine s'est trompé?»). Les pseudo-mots - tous de six lettres - ont été soigneusement construits (12 règles de construction) afin de présenter des items aussi variés que possible, notamment des écarts variables entre les lettres redoublées - de l'absence d'écart (xx) à l'écart maximal (x----x), soit 16 items différents. L'épreuve a été passée par 46 enfants de GS. Dans l'épreuve d'écriture inventée préalable pour sélectionner l'échantillon, aucun des enfants n'a produit de dessin ni pris en compte l'oral dans ses écrits, ces caractéristiques permettant de dire qu'ils se situent à un niveau de développement correspondant à la seconde période de Ferreiro. Les énoncés d'acceptabilité indiquent $34 \%$ de refus quand la même lettre apparaît deux fois dans un même item. Dans le cas de l'absence d'écart, c'est-à-dire quand une lettre apparaît deux fois côte à côte, ce pourcentage de refus s'élève à $39 \%$, et le refus maximum apparaît quand un item donné comporte deux fois des lettres redoublées; dans ce cas, $48 \%$ des enfants estiment que l'item est inacceptable. On voit donc bien se manifester un refus des écrits dans lesquels une même lettre apparaît deux fois, mais ce refus n'a cependant pas le caractère massif auquel on aurait pu s'attendre.

Une autre série de travaux, bien que concernant l'apprentissage implicite et non pas l'hypothèse de variété intra-figurale, apporte néanmoins des données qui intéressent cette question.

Treiman et Cassar (1997) montrent à des enfants anglophones des paires de pseudo-mots comportant des doubles lettres placées au début ou en fin de mot (ex. bbaf / baff), et leur demandent de choisir 
Jacques Fijalkow \& al

celui des deux qui ressemble le plus à un vrai mot. Le matériel présente diverses variantes, tout en s'efforçant de respecter nombre de caractéristiques de l'anglais écrit. L'épreuve est passée par des enfants de la maternelle au collège. En rapport avec notre propos, il apparaît certaines connaissances concernant les lettres doubles. En effet, en maternelle, les enfants préfèrent les doublets en fin de mot (ex. baff) plutôt qu'en début de mot (ex. bbaf), ce qui correspond de fait à une caractéristique de l'anglais écrit. Également, en première année, ils rejettent plus souvent les consonnes redoublées qui n'apparaissent pas dans la langue que celles qui existent réellement. Ces résultats confirment que les enfants sont sensibles à cette propriété de l'écrit, et sont compatibles avec l'hypothèse de variété intrafigurale de l'enfant.

Des travaux ont été effectués avec des enfants français dans la continuité de cette recherche (Pacton, Fayol et Perruchet, 1999; Pacton, Perruchet, Fayol et Cleeremans, 2001). Les tâches, qui consistent à juger et à compléter des non-mots, confirment que ces enfants, dès le $\mathrm{CP}$, sont sensibles à certaines régularités orthographiques tels que la fréquence des doubles consonnes, au fait que les voyelles ne peuvent être redoublées, et à la position légale des doubles consonnes. Dans une première étude des non-mots trisyllabiques sont présentés aux enfants. Ceux-ci comportent un espace à compléter (ex. tubair; u-otir). Les enfants complètent les mots à partir deux étiquettes situées milieu et en bas de la feuille sur lesquelles figure une consonne simple ou double. Le matériel est construit de manière à croiser deux variables: le doublement ou non des consonnes et leur fréquence (ex. consonne simple fréquente/ consonne simple rare; double consonne fréquente/ double consonne inexistante; consonne simple rare/ double consonne inexistante...). Dans une seconde étude, l'expérimentateur présente aux enfants 18 paires de non-mots trisyllabiques (ex. gira-er; iraver) comportant un espace à compléter en position initiale, médiane ou finale. Comme précédemment une consonne simple ou double est présentée en bas des items à compléter $(\mathrm{m} ; \mathrm{ll})$.

Ces résultats paraissent donc, dans le cas du français, compatibles avec ce que Ferreiro exprime en termes d'exigence de variété intrafigurale. Cependant, le fait que les réponses des enfants tiennent compte dès le CP de certaines propriétés de l'anglais ou du français écrit, s'il est compatible avec l'analyse constructiviste de Ferreiro, l'est également avec la conception empiriste selon laquelle l'origine des connaissances vient de l'environnement.

Les résultats de ces diverses recherches convergent donc sur un point : il existe bien chez les jeunes enfants une sensibilité particulière à la répétition d'une lettre donnée dans un même écrit. Ce fait est avéré en italien, en espagnol, en anglais et en français. Toutefois le fait que ces recherches aient été menées avec des dispositifs différents ne permet pas d'aller très au-delà de ce constat. Notons cependant que, quand on s'intéresse non pas à la moyenne mais au nombre d'enfant, les résultats indiquent que le rejet des lettres redoublées n'est le fait que d'un peu plus du tiers d'entre eux. S'il est donc vraisemblable qu'une exigence de variété intrafigurale intervient dans les réponses observées, il se peut néanmoins que ces réponses n'aient pas un caractère universel mais ne concernent qu'un nombre limité d'enfants ${ }^{18}$.

L'exigence formelle de lisibilité, selon Ferreiro, se manifeste encore d'une autre façon.

Un autre procédé utilisé par les enfants est le suivant: essayer de faire correspondre les variations quantitatives dans les représentations à des variations quantitatives dans l'objet référé. Le raisonnement est alors le suivant: les noms des objets plus grands devraient s'écrire avec plus de lettres que ceux des objets petits, de même pour le plus gros, le plus lourd, le plus nombreux, ou le plus âgé. (Ferreiro, 2000, p. 27)

Un dispositif expérimental a été conçu expressément afin d'examiner cette proposition (Bina et Fijalkow, soumis). Dans ce dispositif, l'expérimentateur présente à l'enfant des mots deux à deux sur un écran d'ordinateur, les énonce, répète l'un des deux, puis demande de signaler le mot écrit qui, selon l'enfant, correspond à celui que l'expérimentateur a énoncé. Les mots retenus sont des noms d'animaux comportant pour moitié des mots hétérogènes (à un animal petit correspond un mot long, et à un animal grand un mot court) et pour moitié des mots homogènes (à un animal grand correspond un mot long, et à un animal petit un mot court). 72 enfants de MS et GS ont passé l'épreuve individuellement, à deux reprises dans l'année afin d'examiner la constance des réponses dans le temps. Les résultats globaux sont 
favorables à l'hypothèse quantitative. En effet, lors des deux passations, les choix effectués, tout comme les justifications apportées, sont compatibles avec l'hypothèse quantitative. Mais les résultats montrent aussi que les enfants s'appuient fortement sur la connaissance qu'ils ont des lettres et tout particulièrement de leur nom. La question est également celle de la validité des résultats ainsi obtenus : dans quelle mesure une situation aussi fermée n'induit-elle pas les réponses obtenues? Si tel était le cas, ce dispositif serait créateur d'un artefact plutôt que révélateur d'une hypothèse de l'enfant. De nouvelles études utilisant un matériel voisin (poids, nombre, âge...) dans des conditions moins contraignantes paraissent nécessaires pour en décider.

Le fait que des enfants de 4 et 5 ans apparient plus souvent mot écrit et image selon la taille quand les deux sont congruents que quand ils sont non congruents est également manifeste dans une situation proche de la précédente où ils sont invités tour à tour à dire lequel des deux mots prononcés par l'expérimentateur est le plus long, puis à dire lequel est le plus long quand les deux mots sont présentés par écrit, puis à associer les deux mots aux images qui leur correspondent. Ces résultats ont été obtenus dans un contexte de recherche sur le bilinguisme français-anglais et chinois anglais (Bialystok, 1997), puis anglais-hébreu (Bialystok, Shenfield et Codd, 2000). Ils montrent à nouveau que l'enfant fait bien une relation entre la taille de l'objet et celle du mot dans une situation où la seule issue est de recourir à ce type d'indicateur pour répondre au problème posé. On peut se demander de plus dans quelle mesure le fait d'attirer l'attention de l'enfant sur le critère de longueur ne renforce pas encore cet effet.

Par ailleurs, pour Ferreiro, outre les exigences quantitatives et qualitatives que nous venons d'examiner, estime que

La deuxième période est caractérisée par la construction de modes de différenciation entre les enchaînements de lettres, en jouant alternativement sur les axes de différenciation qualitatifs et quantitatifs. (Ferreiro, 2000, p. 16)

L'analyse des productions écrites par les enfants dans la tâche d'écriture inventée type où l'adulte dicte des mots et/ou des phrases sur une feuille unique montre effectivement, souvent de façon très manifeste, comment l'enfant joue sur le choix des lettres (exigence qualitative) et leur nombre (exigence quantitative) afin de produire un écrit différent pour chacun des énoncés oraux différents qui lui sont proposés. La difficulté toutefois est que, presque toujours semble-t-il, les productions demandées sont effectuées sur une même feuille. Cette particularité de la situation lui permet de se baser sur ce qu'il vient d'écrire pour écrire ce qui lui est demandé maintenant. Il peut donc procéder par autocopie. La probabilité d'une telle procédure est d'autant plus grande que la tâche est difficile pour un enfant dont les connaissances de l'écrit sont par construction insuffisantes par rapport à ce qui lui est demandé. La question est alors de savoir dans quelle mesure les productions recueillies dans ce dispositif résultent d'une authentique exigence formelle liée à un certain niveau psycho-génétique et dans quelle mesure elles résultent de la situation particulière dans laquelle se trouve l'enfant. On peut alors raisonner ainsi : si l'on fait varier la situation d'écriture (l'enfant a ou non la possibilité de s'autocopier) et que ces variations sont sans effet sur l'écriture de l'enfant, on pourra considérer que celle-ci est imputable à son niveau de développement psycho-génétique; dans le cas contraire, si les productions écrites par les enfants changent quand les conditions d'écriture changent, c'est que ces productions résultent en partie de ces conditions et sont donc à imputer à celles-ci et non seulement aux caractéristiques de développement supposées. Dans ce second cas, on pourra penser que, au moins en partie, les caractéristiques de l'écriture observées sont des artefacts expérimentaux, c'est-à-dire des manifestations qui résultent des conditions expérimentales et non des seules caractéristiques du sujet.

L'étude consacrée à cette question (Pomel, 2008) a été effectuée sur un échantillon de 117 jeunes enfants sélectionnés à l'issue d'un prétest (écriture de 4 mots) : ont été retenus les enfants qui effectuent un traitement visuel majoritaire de l'écrit (peu de rapports entre ce qu'ils écrivent et l'oral) et utilisent principalement des lettres conventionnelles. Ces enfants sont alors invités à écrire en petit groupe, l'observateur étant présent, 4 mots bisyllabiques (cochon, cheval, poisson, lapin). Le dispositif comporte trois situations expérimentales : l'écriture d'un mot par page (ce qui exclut l'autocopie), l'écriture en liste (supposée la favoriser) et l'écriture en 
ligne (supposée plus neutre, n'interdisant ni ne facilitant a priori l'autocopie). Chaque enfant effectue deux des trois tâches, à quelques jours de distance : le premier groupe écrit les mots en liste (les uns en dessous des autres), puis en ligne (les uns à côté des autres); le second groupe écrit les mots en liste, puis un mot par page; le troisième groupe écrit un mot par page, puis en ligne. Les résultats montrent que les enfants n'utilisent pas les mêmes procédés de variation qualitative et quantitative selon la tâche. En particulier, quand l'enfant écrit en liste, le voisinage visuel des graphies précédentes l'incite à s'autocopier, c'est-à-dire qu'il reprend les lettres ou les suites de lettres qui sont dans son champ de vision, et/ou utilise la première graphie comme unité de mesure (pour produire la même quantité, une quantité plus grande ou plus petite, voire en alternance).L'enfant n'est pas forcément conscient de ce comportement qui semble simplement l'aider à écrire. Toutefois, quand il cherche manifestement à différencier les graphies, l'autocopie semble lui faciliter l'opération. Il reprend alors aisément des suites de lettres en variant leur position et se préoccupe moins de variation quantitative, produisant des graphies plus courtes et plus homogènes. En l'absence de voisinage visuel, il est incité à rechercher de nouvelles lettres et les variations qualitative et quantitative sont davantage contrastées.

Il se confirme ainsi de façon massive que les variations interfigurales énoncées par Ferreiro apparaissent essentiellement dans la situation d'écriture en liste. Dans ce cas en effet on observe le même nombre de lettres ou les mêmes lettres mais ordonnées différemment, la proximité visuelle fournissant alors à l'enfant un moyen facile pour résoudre la tâche difficile qui lui est demandée. Le fait que les modes de différenciation indiqués apparaissent essentiellement dans cette situation et que les mêmes enfants procèdent de manière différente quand la situation n'est plus la même interroge donc sur les rôles respectifs qui reviennent au niveau de développement de l'enfant et à la situation d'écriture. Il se peut en effet que ce qui a été interprété jusqu'ici en termes d'articulation entre des exigences quantitatives et qualitatives propres à l'enfant se révèle être limité à une tâche particulière plutôt qu'à la pensée de l'enfant en général. Une fois encore, les faits confirment donc l'existence des modalités de réponse identifiées par Ferreiro, mais celles-ci apparaissent à l'issue de cette expérience, fortement dépendantes de la situation d'écriture. On peut se demander dès lors s'il convient de leur accorder autant de place dans les conceptualisations de l'enfant que celle qui leur a été attribuée jusqu'ici. À nouveau, le dispositif expérimental paraît contribuer aux résultats observés, ce qui appelle à de nouvelles recherches.

La troisième période

Ferreiro présente ainsi la troisième période :

Une hypothèse nouvelle fait alors son apparition, à savoir que le nom prononcé est décomposable en parties, en "petits bouts" les uns à la suite des autres, tout comme le nom écrit est un composé de parties placées dans un certain ordre. Une nouvelle correspondance terme à terme est dès lors possible entre ces deux ensembles ordonnés. C'est le début de la phonétisation de l'écriture. Les "petits bouts " que l'enfant trouve en décomposant le mot sont bien entendu les syllabes ». (Ferreiro, 2000, p. 45)

Au-delà même de cette hypothèse, la syllabe apparaît en vérité comme le pivot de la psychogenèse. De façon constante en effet, des travaux les plus anciens (Ferreiro et Teberosky, 1979) aux plus récents Ferreiro (Ferreiro et Gomez-Palacio, 1988; Vernon et Ferreiro, 1999; Ferreiro, 2009), les auteurs distinguent quatre niveaux : pré-syllabique, syllabique, syllabico-alphabétique, alphabétique. On voit donc que c'est en référence à la syllabe - ce qui est avant, pendant, après - qu'est conçue la psychogenèse de l'écriture chez l'enfant. En centrant l'analyse sur ce que nous appellerons dès lors écriture unitaire (une syllabe = une lettre), la question est de savoir, chez les enfants français, quelle est l'importance de cette réponse dans l'ensemble des productions des enfants, dans quelle mesure elle est exclusive, et si elle est aussi uniforme et simple que ce que l'on pourrait supposer.

Deux analyses d'un corpus (Pasa, Creuzet et Fijalkow, 2006; Creuzet et Pasa, 2009) ont été effectuées. L'échantillon de départ comporte 144 enfants de GS (5-6 ans), dont seuls 115 ont été conservés à l'issue d'un test préalable montrant une prise en compte manifeste de l'oral dans leur écriture. À ces 115 enfants, on demande alors individuellement d'écrire six phrases contenant chacune deux mots tests dans lesquels apparaissent les trois types de structures syllabiques les plus courantes en français : 
$\mathrm{CV}, \mathrm{CCV}$ et $\mathrm{CVC}^{19}$. Les productions écrites sont suivies d'un entretien métagraphique destiné à savoir comment l'enfant a codé les syllabes cibles.

L'analyse, qui met en relation chaque syllabe cible avec le nombre de lettres qui y correspondent, fait alors apparaître non pas un mais quatre types d'écriture pour représenter les syllabes cibles :

- $\quad$ écriture unitaire : une seule lettre est utilisée pour transcrire une syllabe,

- $\quad$ écriture duelle : deux lettres sont utilisées pour les syllabes qui en nécessitent trois (les syllabes CCV et CVC),

- $\quad$ écriture complète : autant de lettres utilisées que de phonèmes à coder (deux pour les syllabes CV, trois pour les syllabes CCV et CVC),

- $\quad$ écriture excédentaire : plus de lettres utilisées que de phonèmes à coder (trois ou plus pour les syllabes $C V$, quatre ou plus pour les syllabes $C C V$ et (VC).

Si l'on considère la totalité du corpus, les résultats montrent que, de façon générale, l'écriture unitaire l'emporte sur toute autre modalité d'écriture (65\% des syllabes produites). Les enfants de GS utilisent préférentiellement une lettre pour coder une syllabe. Cette modalité d'écriture, si elle n'est pas la seule, est néanmoins majoritaire dans leurs productions.

Examinant ensuite spécifiquement les enfants qui écrivent exclusivement de façon unitaire, on s'aperçoit que seuls 50 enfants (43\%) présentent une écriture unitaire, tandis que les 65 autres (57\%) utilisent deux types de traitements ou plus. Moins d'un enfant sur deux procède donc exclusivement à une écriture unitaire.

On se propose alors de voir comment procèdent les enfants en fonction de la structure de la syllabe. Cette analyse fait apparaître que le type d'écriture est fonction de la structure de la syllabe à écrire. Ainsi, les enfants codent plus volontiers une lettre par syllabe pour les structures CV (80 \%) et CCV (75\%) que pour la structure CVC (55\%). Il est donc vraisemblable que la façon dont l'enfant conceptualise la relation entre l'oral et l'écrit ne suffit pas à expliquer ces réponses, mais que des facteurs linguistiques entrent en jeu qui favorisent par exemple le traitement syllabique dans le cas des structures ouvertes - CV et CCV (se terminant par une voyelle) - et y font obstacle quand la syllabe est fermée - de type CVC (se terminant par une consonne).

Si l'on examine enfin à quoi correspond véritablement le fait d'écrire une lettre par syllabe, l'entretien métagraphique apporte d'intéressantes précisions : il permet en effet de dépasser les apparences qui laissent penser que, quand le nombre de lettres écrites correspond au nombre de syllabes, l'enfant procède à partir d'une hypothèse syllabique. Ce que permet de voir l'entretien c'est qu'une réponse unitaire peut résulter de deux types de traitements distincts : un traitement syllabique (pour l'enfant, une lettre suffit à traduire une syllabe) ou un traitement phonémique (l'enfant écrit une lettre pour un phonème qu'il discrimine). Dans ce dernier cas, on dira que l'écrit est partiellement phonémique car l'enfant ne code dans une syllabe que le phonème qu'il parvient à percevoir, tout en sachant pertinemment qu'il ne code qu'une partie de l'énoncé. L'enfant dit alors souvent, qu'il entend autre chose mais qu'il ne sait pas l'écrire. L'écriture unitaire apparaît donc dans de tels cas être non pas syllabique mais phonémique (un phonème discriminé par syllabe) et partielle (un seul phonème par syllabe au lieu de deux).

Par exemple, Jade écrit les lettres OI pour Tèmi, le prénom de la fourmi.

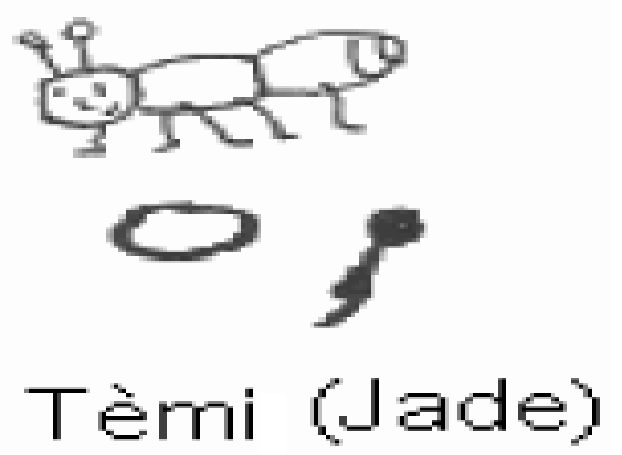

Figure 6 - Traitement phonémique partiel

La lettre $O$ traduit apparemment la syllabe [t $\mathbb{\nabla}]$ et la lettre I traduit la syllabe [mi]. Or, l'entretien montre que, contrairement à ce que l'on pourrait 
Jacques Fijalkow \& al

supposer, Jade n'effectue pas un codage syllabique;

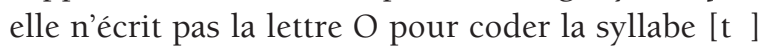
mais pour représenter le phonème $[\bigotimes]$, dont elle ne connaît pas par ailleurs la traduction graphique :

«Un $[\otimes]$, je connais pas la lettre [...] Je connais que ça. [...] C'est comment?»

Si le traitement opéré ici est sans doute syllabique à l'oral au départ (l'enfant sépare les deux syllabes), il ne l'est pas à l'écrit à l'arrivée. En effet, dans chaque syllabe, l'enfant procède à une analyse à l'issue de laquelle il distingue un seul phonème car il ne parvient pas à discriminer les deux phonèmes de chaque syllabe. Son écriture est phonémique partielle, faute de parvenir à être exhaustive.

De manière générale, le traitement syllabique est le plus utilisé pour coder les syllabes cibles. En effet, $41 \%$ des réponses renvoient à un traitement syllabique contre $26 \%$ à un traitement phonémique partiel, $24 \%$ à un traitement phonémique pluriel (l'enfant effectue une analyse phonique de l'énoncé et réussit à extraire deux des trois phonèmes qui constituent la syllabe) et seulement $9 \%$ à un traitement exhaustif.

En bref, les analyses réalisées, si elles confirment la place importante de l'écriture unitaire dans les productions des enfants, montrent cependant qu'elle n'est exclusive que pour un peu moins de la moitié des enfants. Elles montrent en outre que ce type de réponse n'est pas uniforme mais varie en fonction de la structure de la syllabe considérée et qu'il est donc nécessaire de prendre en compte ce type de variable linguistique ${ }^{20}$. Elles montrent enfin que les réponses unitaires ne correspondent pas nécessairement à une hypothèse syllabique.

Une autre étude, réalisée au Québec, interroge fortement la place à réserver à la syllabe dans le développement de l'écriture des enfants francophones. Bégin, Saint Laurent et Giasson (2005) y examinent l'évolution de l'écriture inventée en rapport avec la conscience phonologique et l'orthographe lexicale. L'étude, longitudinale, débute avec 433 enfants en $1^{\text {ère }}$ année, se poursuit avec 338 en $2^{\mathrm{e}}$ année, 204 en $3^{e}$ année et 223 en $4^{e}$ année ${ }^{21}$. Trois épreuves d'écriture inventée, de conscience phonologique et d'écriture de textes permettent d'apporter des éléments de réponse aux trois objectifs de l'étude (description des écritures provisoires, lien avec la conscience phonologique et apport des écritures provisoires pour l'orthographe lexicale). La tâche d'écriture consiste à faire écrire aux enfants cinq noms d'animaux (girafe, abeille, escargot, hibou et éléphant). L'évaluation se fait en fonction du nombre de phonèmes représentés. Les résultats des enfants de première année montrent que certains niveaux indiqués par Ferreiro et GomezPalacio (1988) se retrouvent dans les productions des élèves québécois, mais que leur répartition dans ces niveaux ne correspond pas à celle obtenue par Ferreiro et Gomez-Palacio ( $8 \%$ vs $90 \%$ pour les écritures pré-syllabiques, $87,9 \%$ vs $3 \%$ syllabicoalphabétiques, et 3,2\% vs $3 \%$ alphabétiques). Cette différence peut être imputée à la scolarisation plus précoce des enfants québécois. Le fait le plus intéressant pour notre propos est autre : les auteures indiquent que les productions syllabiques signalées par Ferreiro sont absentes des productions des enfants québécois. La question de la place de la syllabe dans le développement de l'écriture inventée chez l'enfant se pose donc posée à partir de cette recherche québécoise de façon plus radicale encore qu'à partir des recherches françaises.

Centrer le développement sur la syllabe pose d'autres problèmes concernant l'évolution de l'écriture inventée. Dans le cas le plus fréquent en effet, si la référence à la syllabe apporte un éclairage certain sur l'écriture des mots, le peu de cas fait à la phrase met en cause la généralité de conclusions qui reposent essentiellement sur l'analyse de mots. L'écriture de la phrase apporte pourtant de précieuses informations. Ainsi, au niveau dit pré-syllabique, avant même qu'apparaisse donc dans les productions quelque correspondance que ce soit avec une syllabe ou un phonème, de nombreux enfants écrivent davantage de lettres pour écrire une phrase que pour écrire un mot (Fijalkow et Liva, 1993). Un tel comportement indique qu'ils commencent à établir un lien avec le continuum sonore, et ce fait s'accommode mal d'une caractérisation de la période comme l'absence de toute recherche de correspondance entre écrit et oral.

Un autre exemple est celui des «écritures différenciées avec valeur sonore initiale "(Ferreiro et Gomez-Palacio, 1988, p. 82), qui correspond à l'écriture d'une quantité de lettres qui ne semble pas déterminée par un essai de correspondance sonore, 
mais dont l'attaque ( $1^{\text {ère }}$ lettre) est une des lettres qui correspond à la valeur sonore de la $1^{\text {ère }}$ syllabe du mot. Ici encore, la prise en compte du phonème est manifeste et on peut craindre à nouveau que la prééminence de principe accordée à la syllabe ne conduise à minimiser les efforts de la prise en compte de l'oral par les enfants.

Un dernier exemple concerne encore la phrase, cette fois dans la période dite syllabique. Ferreiro observe que les enfants écrivent alors soit une lettre par syllabe, soit une lettre par mot. On voit combien l'étiquette syllabique donnée à la période, s'accommode mal, dans le second cas, d'une écriture que l'on pourrait tout aussi bien caractériser de lexicale (un mot $=$ une lettre). En bref, cette façon de focaliser le développement de l'écriture autour d'une seule unité (la syllabe), alors que certaines productions rendent compte du travail de l'enfant avec d'autres unités (mot, phonème, phrase), sous son apparente clarté, risque d'empêcher de percevoir que l'enfant analyse très tôt l'oral au-delà de la seule syllabe.

\section{Les aspects techniques de l'entretien avec l'enfant}

L'analyse présentée plus haut (Pasa, Creuzet, Fijalkow, 2006; Creuzet et Pasa, 2009) incite à s'intéresser aux aspects techniques de l'entretien avec l'enfant. Ainsi, si dans l'étude citée l'entretien permet de mieux différencier ce qui est réellement syllabique et ce qui ne l'est pas, à l'inverse, l'entretien avec l'enfant peut être source de confusions. Tel est le cas de l'activité au moyen de laquelle la procédure suivie par l'enfant est identifiée : en relecture ou en écriture. Il peut advenir en effet que des observations faites en relecture soient malencontreusement rapportées à l'écriture. Prenons un exemple d'analyse issue du corpus de Ferreiro, la production d'Alma Rosa, qui est entièrement interprétée sur les bases de la relecture effectuée après écriture :

« La correspondance sonore entre lettres et syllabes se fait parfaitement pour les mots à 3 ou 4 syllabes, mais l'exigence de quantité minimale affecte les mots mono et bisyllabiques, auxquels on ajoute plus de lettres que ce qui leur revient (et même plus de lettres que le minimum exigé par les enfants eux-mêmes). Dans ce cas, 3 lettres sont la quantité minimale, mais, aussi bien le monosyllabe que le mot bisyllabique ont plus de trois lettres : c'est pourquoi nous parlons d'une forte exigence quantitative. La valeur sonore conventionnelle n'apparaît que dans un mot (« mariposa ») .»(Ferreiro, 1988, p. 88)

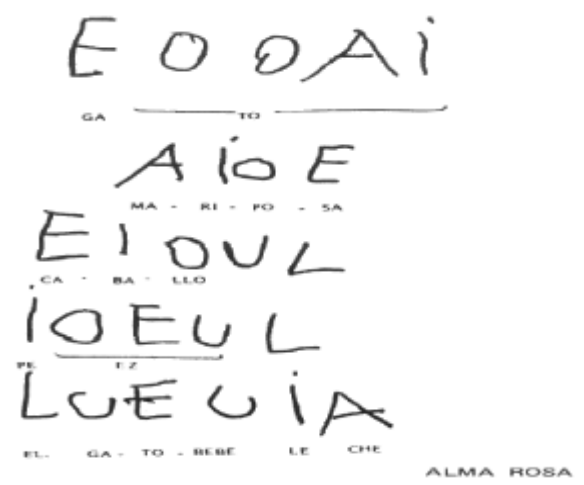

Figure 7 - Alma Rosa, écriture syllabique

Or la procédure syllabique d'écriture (une lettre par syllabe) n'est dans ce protocole avérée que pour un seul mot, mariposa. Pour tous les autres mots, il pourrait s'agir d'une procédure syllabique de relecture. Il est d'ailleurs possible que l'écriture syllabique du mot mariposa soit un effet temporaire induit par la demande de relecture de la première graphie gato pour laquelle l'enfant recourt à un procédé de lecture syllabique.

En 2000, Ferreiro reconsidère ce point en précisant que les étapes ne sont pas franchies au même moment en lecture et en écriture et que l'hypothèse syllabique ne sert au début qu'à justifier une production qui n'a pas été guidée par elle. Cependant, rejetant la distinction traditionnelle entre lecture et écriture, Ferreiro considère que les productions écrites des enfants sont "révélatrices des niveaux de conceptualisations $»(2000$, pp. 15-16). De ce fait, que l'enfant puisse interpréter différemment l'écrit selon qu'il l'observe ou qu'il le produit, revêt une importance toute relative aux yeux de l'auteure. Or, que le jeune enfant possède des compétences de syllabation à l'oral et les utilise facilement pour lire l'écrit, ne doit pas nous autoriser à en déduire la façon dont il le produit. Les progrès de la recherche en écriture inventée nous paraissent donc passer par la mise au point de procédures standardisées de l'entretien avec l'enfant. Elles conditionnent sans doute la possibilité de réaliser des réplications et en général des comparaisons rigoureuses. 
Jacques Fijalkow \& al

\section{Le tournant socioconstructiviste}

Rappelons sommairement que, suivant la perspective piagétienne qui est celle de Ferreiro, la langue écrite est considérée comme un développement plutôt que comme un apprentissage. Ceci signifie en particulier que la progression de l'enfant trouve sa dynamique dans des processus internes au sujet plutôt que dans des facteurs externes. Ferreiro parle de "relative indépendance de la psychogenèse par rapport aux interventions directes du milieu »(Ferreiro, 1988). Si l'on met en doute cette « relative indépendance " la question se pose alors d'une troisième voie entre l'empirisme radical et le constructivisme universel, une voie socio-constructiviste. En d'autres termes, étant entendu que l'entrée dans l'écrit est de nature psycho-génétique, la question est de savoir si ce processus est unique ou s'il peut prendre des formes variables en fonction du contexte, didactique ou linguistique. Si tel était le cas le processus étudié pourrait alors être qualifié non plus de psycho-génétique mais de psycho-socio-génétique.

Que le contexte linguistique puisse jouer apparaît dans les publications les plus récentes (Ferreiro, 1998, p. 16), mais pas le contexte didactique, sinon que Ferreiro choisit de prendre «la plus grande quantité possible d'écoles et, à l'intérieur, la plus grande quantité possible de classes » de façon à minimiser, à travers cette «diversité, le poids de cette variable non contrôlée "(Ferreiro, 1988, p. 4). Une lecture attentive montre cependant que les données analysées sont produites par des enfants exposés à un enseignement présentant « d'emblée les principes de base du système alphabétique »(id, p. 58), et les analyses rapportées font état de nombreuses remarques qui laissent supposer que la plupart de ces contextes sont de type traditionnel et relèvent d'une méthode synthétique, c'est-à-dire basé sur la syllabe. Les enfants observés par Ferreiro ont un profil très particulier : n'ayant pas été préscolarisés en un lieu où des activités d'exploration de l'écrit sont communément proposées, ils sont directement confrontés à l'acquisition de la combinatoire. Cette entrée directe dans l'écrit n'est pas un facteur négligeable et invite à s'interroger sur les réponses d'enfants placés dans d'autres conditions didactiques. Cette circonstance est à considérer particulièrement quand on considère le fait que les données de l'étude principale (Ferreiro et Gomez-Palacio, 1988) ont été recueillies tout au long de la première année d'école élémentaire au Mexique dans un contexte linguistique (espagnol) et didactique (méthode synthétique) particulier.

\section{Études comparatives dans des classes à didactique différente}

Une première étude comparative (Pasa, 2002a) examine l'influence de deux contextes didactiques contrastés. Le but est de repérer, à travers l'écriture inventée, la manière dont deux groupes de 11 enfants de CP formalisent les relations oral-écrit en fonction du travail didactique effectué en classe. Dans la première classe, l'enseignant utilise une méthode mixte à dominante phonétique dont la progression est définie par l'ordre de présentation des CGP dans un manuel privilégiant les unités courtes en début d'apprentissage (graphèmes d'une lettre, syllabes de type CV, mots mono ou bisyllabiques, phrases minimales...). Dans la seconde classe, l'enseignant pratique une approche langage entier faisant appel à des textes authentiques dont les structures linguistiques présentent la complexité de la langue. L'évaluation consiste à faire écrire aux enfants, 4 fois dans l'année, 6 mots cibles faisant varier les structures syllabiques (syllabes CV vs non $\mathrm{CV}$ ) et graphémiques (graphèmes simples uniquement vs graphèmes composés), ainsi que l'enseignement ou non des CGP présentes dans les mots (pour la classe phonique). Les résultats montrent un effet du contexte didactique dans la diversité des stratégies mises en œuvre dans l'encodage graphique (par exemple, l'utilisation du nom des lettres ou de mots connus pour coder une syllabe apparaît majoritairement dans la classe langage entier). De plus, certaines difficultés (liées à la polyvalence graphique, aux similitudes phonographiques visuelles ou auditives, à l'absence de relation biunivoque entre phonie et graphie...) semblent directement liées à la progression hiérarchisée d'un enseignement focalisé sur la présentation du code phonographique.

Une seconde recherche comparative menée dans des contextes scolaires contrastés (Pasa, 2002b) étudie, durant une année scolaire, les comportements de 66 apprentis-scripteurs issus de trois classes de $\mathrm{CP}$. Les trois enseignantes sont comparables quant à leur âge, leur formation et leur expérience professionnelle, mais diffèrent dans leur façon d'aborder 
l'enseignement de la langue écrite (didactique classique centrée sur l'enseignement du code phonographique à partir d'un manuel de lecture; didactique de type langage entier centrée sur la dimension sémantique de l'écrit; approche socio-culturelle du langage écrit privilégiant la découverte des aspects fonctionnels et communicationnels, les écrits sociaux dans des situations d'interactions sociales). Les résultats montrent des variations interclasses dans les représentations élaborées par les enfants, dans leurs découvertes successives des propriétés du système d'écriture et dans leurs compétences phonologiques, qui induisent des progressions différentes. Les élèves exposés à une didactique centrée sur les CGP sont rapidement capables de traduire la forme orale des mots (en particulier si les règles phonographiques nécessaires leur ont déjà été enseignées; dans le cas contraire ils se révèlent plus faibles que les autres), mais ce sont eux qui rencontrent le plus de difficultés liées aux similitudes phoniques ou graphiques. En outre, leurs progrès en orthographe sont moindres que ceux des élèves des deux autres classes. Ces derniers semblent se construire d'emblée une vision plus complète du système d'écriture, incluant à la fois des unités visant à transcrire les phonèmes, les phonogrammes, et des unités qui traduisent une information sémiographique et qui sont inaudibles, comme les morphogrammes (ceci est en outre confirmé dans une autre étude auprès de deux échantillons français et québécois (Pasa et Morin, 2007)). Enfin, des effets statistiques différentiels des facteurs linguistiques sont observés. C'est dans la classe où l'enseignement phonographique est le moins intensif et directif (approche socioculturelle), que les élèves sont les moins sensibles aux difficultés graphémiques (graphème simple vs graphème composés) ou polygraphiques (phonèmes ayant plusieurs transcriptions possibles), ce qui tend à montrer que, du point de vue de l'enfant, les correspondances phonographiques ne sont pas hiérarchisables en termes de plus ou moins grande complexité. En revanche, le développement des compétences phonologiques est plus lent dans cette classe et l'écriture est plus largement soumise aux variations syllabiques, certaines structures (CVC, CCV ou (VC) étant plus difficiles à analyser. Enfin, c'est dans cette classe que les stratégies de codage syllabique au moyen d'un nom de lettre ou d'un morphème connu perdurent le plus longtemps dans l'année.

\section{Études de didactique expérimentale}

Une autre façon d'examiner le rôle du contexte didactique est de nature expérimentale. Dans un cadre de didactique expérimentale, le souci de faire apparaître l'effet d'éventuelles variables didactiques conduit à abandonner la position classique d'observateur d'un développement qui se déroule en amont pour une position consistant à tenter d'intervenir sur ce développement même. En effet, si l'on effectue une didactique expérimentale, la situation de recherche n'est alors plus une situation où l'on suscite, ponctuellement, la manifestation d'un développement extérieur à celle-ci, mais une situation où l'on tente de faire évoluer les réponses de l'enfant au cours de séances répétées. Le chercheur est alors moins observateur qu'acteur.

Toutes ces études ont été menées en MS ou GS. Le plan expérimental utilisé le plus souvent comporte deux à trois groupes d'enfants appariés suivant des critères spécifiques, c'est-à-dire relatifs à l'aspect de l'écriture inventée sur lequel on se propose d'intervenir dans l'expérience. Ces enfants passent un prétest et un post-test d'écriture inventée (variable dépendante) et sont soumis dans l'intervalle à une intervention didactique qui varie d'un groupe à l'autre (variable indépendante). On dispose ainsi d'un groupe expérimental et d'un groupe témoin, ou de plusieurs groupes expérimentaux et d'un groupe témoin. Dans ce dernier cas, l'intervention didactique vise à reproduire dans un groupe les pratiques pédagogiques habituelles dans les classes et, dans l'autre, à proposer une pédagogie plus innovante. Chaque groupe comporte environ 10 enfants, soit 20 à 30 enfants par expérience.

Comme on l'a vu plus haut, une intervention expérimentale faisant varier la quantité de lecture et d'écriture a des effets significatifs sur la production de dessins en réponse à une demande d'écriture (Sarris, 1996). Le contexte didactique apparaît déterminant : plus les enfants travaillent avec l'écrit, moins ils utilisent le dessin en situation d'écriture inventée.

On l'a vu également, à un certain moment les enfants utilisent préférentiellement les lettres de leur prénom pour écrire, quel que soit ce qu'on leur demande d'écrire. La didactique peut-elle avoir un effet sur ce paramètre? Pour le savoir, des enfants de GS ( $N=28)$ sont divisés en deux groupes appariés par la classe, l'âge, le sexe et un critère spécifique: 
Jacques Fijalkow \& al

l'utilisation majoritaire des lettres de leur prénom dans le prétest (plus de la moitié des lettres du prénom utilisées au moins une fois) (Sarris, 1996). À un groupe d'enfants, on enseigne alors à écrire leur nom de famille, tandis qu'on continue à faire écrire leur prénom aux enfants de l'autre groupe, et qu'un troisième groupe (témoin) demeure en classe. L'entraînement dure 12 séances.

L'analyse des réponses distingue trois types de lettres: lettres du prénom / lettres du nom / autres lettres. La comparaison des résultats entre prétest et post-test pour chaque groupe, ainsi qu'entre les groupes au post-test - soit 9 hypothèses examinées au total - montre que lorsqu'on fournit de nouvelles ressources littérales aux enfants, en l'occurrence ici les lettres de leur nom de famille, leurs productions écrites sont plus riches que celles des enfants qui n'ont à leur disposition que les lettres de leur prénom et plus riches encore que celles de ceux qui n'ont pas bénéficié d'un entraînement supplémentaire. Ceci invite à considérer que l'utilisation fréquente des lettres du prénom dans les productions écrites habituelles des enfants en situation d'écriture inventée peut être l'effet d'un contexte éducatif dans lequel le prénom est le mot dont l'écriture est privilégiée par l'environnement.

Une autre caractéristique des productions des enfants en situation d'écriture inventée concerne la position de leurs écrits dans la page. Quand les enfants disposent d'une seule feuille de papier, on observe qu'ils écrivent le plus souvent les mots les uns en dessous des autres en haut et à gauche de la page (écriture en liste) et les phrases en plaçant les mots à la suite les uns des autres de gauche à droite (écriture en ligne). Ces conventions d'écriture, qui ne font généralement pas l'objet d'un enseignement explicite, sont mises en œuvre par les enfants de manière variable. Dans quelle mesure peuvent-elles être acquises à l'issue d'un enseignement spécifique?

L'expérience (Sarris, 1996) est effectuée avec 24 enfants de GS répartis en 3 groupes appariés selon la classe, l'âge, le sexe, le milieu social et un critère spécifique à la tâche: le fait d'utiliser la page de manière non conventionnelle au prétest.

On s'efforce d'enseigner à écrire de manière conventionnelle à un premier groupe : lors de chaque séance, l'adulte lit d'abord une histoire à l'enfant, puis l'adulte écrit sous la dictée de l'enfant (dictée à l'adulte) une liste de mots et une phrase, en indiquant les normes d'écriture correspondantes (écriture en liste ou en ligne); enfin il inverse les rôles en dictant à deux reprises les mêmes éléments (mots et phrases) à l'enfant, d'abord en l'invitant à s'auto-répéter le matériel à écrire à haute voix (auto-langage), puis sans oralisation audible. Avec le second groupe, l'entraînement consiste à lire l'histoire à l'enfant puis à l'inviter à écrire les mêmes mots et phrases que dans le premier groupe, après lui avoir indiqué les normes d'écriture correspondantes (écriture en liste ou en ligne) et avoir placé une gommette au point de départ de l'écriture. Dans cette seconde condition, les enfants écrivent directement, en une seule fois et sans être passés par une dictée à l'adulte. Le troisième groupe (témoin) demeure dans la classe. L'entraînement dure 11 séances.

L'analyse des résultats repose sur l'utilisation d'une grille d'analyse des mots (6 échelons : des mots au centre et en ligne jusqu'aux mots en haut et à gauche) et d'une autre grille pour analyser les phrases (6 échelons : de la phrase écrite au milieu de la page et en colonne à la phrase écrite en haut et à gauche et en ligne). La comparaison des résultats entre prétest et post-test pour chaque groupe, ainsi qu'entre les trois groupes au post-test, montre que les hypothèses sont globalement vérifiées : il est possible d'enseigner les normes d'écriture des listes de mots et cet enseignement est plus ou moins efficace suivant les conditions pédagogiques mises en œuvre.

Que se passe-t-il si, au lieu d'intervenir sur le nombre de lettres, on agit maintenant sur leur forme? Peut-on faciliter la production écrite de l'enfant en lui faisant utiliser tel ou tel type de caractères? Considérant que la connaissance qu'ont les enfants des lettres qu'ils utilisent est plus de nature figurative que cognitive, on peut penser qu'intervenir sur leur forme graphique pourrait avoir un effet facilitateur ou inhibiteur selon les conditions expérimentales.

L'expérience (Cazes, 1996) est effectuée avec 20 enfants de MS répartis en 4 groupes appariés par la classe, l'âge, le sexe, le milieu social et le niveau de traitement de l'écrit au prétest d'écriture inventée : tous effectuent un traitement figuratif ou visuel. L'expérience consiste alors à entraîner l'enfant, lors de chaque séance, à écrire son prénom ainsi qu'un 
mot issu de l'histoire qu'on vient de lui lire (un mot différent d'une séance à l'autre) mais, suivant le groupe dans lequel il se trouve, on l'entraîne soit à utiliser la même police de caractères pour son prénom et pour l'autre mot à écrire (effet facilitateur attendu), soit à utiliser une police de caractères différente pour son prénom et pour l'autre mot (effet inhibiteur attendu).

De manière plus précise, le plan comporte quatre groupes expérimentaux: deux groupes avec des lettres homogènes (prénom en capitales / autre mot en capitales; prénom en script / autre mot en script) et deux groupes avec des lettres hétérogènes (prénom en capitales / autre mot en script; prénom en script / autre mot en capitales). L'entraînement s'est étendu sur 8 séances.

L'analyse des résultats porte sur la position des enfants dans une grille de développement de l'écriture inventée (Fijalkow et Liva, 1993), sur le nombre de lettres différentes utilisées et sur leur origine (lettres du prénom / autres lettres). La comparaison des résultats entre prétest et post-test pour chaque groupe, ainsi qu'entre les groupes au post-test, montre que globalement les hypothèses émises sont vérifiées: les enfants entraînés à utiliser les mêmes caractères produisent au post-test des protocoles plus évolués et plus riches qu'au prétest ou que ceux des groupes entraînés à utiliser des caractères hétérogènes : leurs productions se situent à un niveau plus avancé de la grille, comportent plus de lettres, et ceci tient aussi bien à une plus grande utilisation des lettres du prénom qu'à l'utilisation de lettres nouvelles (extension du répertoire). Ainsi, quand on renforce la forme graphique en faisant écrire le prénom et des mots avec une même police de caractères, on observe un meilleur développement et un enrichissement de l'écriture par rapport au cas où les caractères sont différents pour les deux types de mots ${ }^{22}$. On voit donc qu'une intervention didactique sur la forme des mots a un effet sur les productions des enfants en situation d'écriture inventée.

L'écriture inventée de la phrase est beaucoup moins étudiée que celle du mot. Son analyse pose des problèmes différents, celui notamment de la segmentation de la phrase en différents constituants ou encore des unités d'oral que l'enfant représente à l'écrit (Farré, 2000). S'agissant d'un objet peu connu, dans un premier temps, la recherche consiste à identifier les procédés de segmentation de la phrase, puis à recourir à la didactique expérimentale. La première étude porte sur 42 enfants de MS et GS. Les phrases dictées individuellement aux enfants comportent un, deux ou trois noms et un verbe (ex. le lapin grignote, le lapin et la souris grignotent, le lapin, la souris et le rat grignotent). Après écriture, l'adulte demande des tâches de signalement (ex. Montre-moi où est écrit lapin) et de verbalisation (ex. Qu'est-ce qui est écrit $i c i$ ?). L'analyse montre que certaines productions ne sont pas segmentées et que tous les segments d'oral ne sont pas écrits. De plus, parmi les segments écrits, l'auteur relève des écrits clairement segmentés en mots ou groupes de mots et où les CGP sont quasiment inexistantes. Ce résultat permet de voir que la mise en relation de l'oral et de l'écrit commence parfois à un niveau d'unités plus larges que les CGP.

Enfin, une intervention expérimentale (Farré et Fijalkow, 2009) est effectuée afin de voir s'il est possible d'améliorer la segmentation de la phrase et si cette amélioration est fonction de la nature de l'intervention. 30 enfants de GS en ZEP sont répartis en trois groupes et soumis à une intervention portant sur 6 phrases de structures syntaxiques différentes dictées tour à tour par l'adulte à l'enfant et par l'enfant à l'adulte. Dans le groupe 1, l'intervention est centrée sur le mot, dans le groupe 2 sur le phonème, tandis que le groupe 3 est témoin. Avant intervention, à l'écrit, la majorité des enfants segmentent les phrases de façon non conventionnelle, sous-segmentent ou sur-segmentent; à l'oral, ils segmentent de façon non conventionnelle ou sous-segmentent. Les résultats montrent que la segmentation évolue suite à l'intervention et qu'elle varie suivant l'unité linguistique sur laquelle on met l'accent.

Pomel (2008) s'intéresse au moment où l'enfant de 4-6 ans appréhende l'écrit comme du langage et fait donc usage de ce dernier en lecture/écriture. Les résultats de cette recherche non seulement éclairent sur la nature et le rôle des paroles que l'enfant s'adresse en cours d'écriture, mais elle montre l'intérêt de ce langage et de l'enseignement de sa mobilisation pour l'enfant qui ne le fait pas. Il s'agit en effet d'autolangage, une compétence langagière à rapprocher du langage intérieur, commune à tout être humain qui l'utilise pour s'entendre réfléchir et réguler son action. Nombre de chercheurs s'intéressent à ce langage pour 
Jacques Fijalkow \& al

soi. Le béhavioriste, attentif aux manifestations physiques de ce langage, peut en déduire sa parenté avec la pensée. Le cognitiviste, le percevant comme un indicateur de l'activité cognitive, l'observe à travers des tâches cognitives diverses, comme le rappel d'images ou la lecture de texte, et peut mettre en évidence les relations de l'auto-langage avec la complexité de la tâche et la compréhension, ainsi que sa fonction de maintien en mémoire de l'information. Le constructiviste, préoccupé par les outils cognitifs que construit l'enfant procède à une observation clinique de l'enfant auto-langagiant. Ce faisant, il étudie le développement de l'auto-langage et peut remarquer les conditions intrapsychologiques de sa mobilisation et de son efficience. Le socioconstructiviste l'observe in situ dans les conditions sociales de sa mobilisation : les interactions. Il prend en compte la nature sociale de l'auto-langage et peut alors décrire le processus d'appropriation par l'enfant et identifier l'importance des variables environnementales sur ce comportement (contexte familial et contexte scolaire). Cependant, ces différentes approches ne dépassent pas les limites $\mathrm{du}$ constat. Pour aller plus loin une intervention expérimentale est réalisée.

38 enfants de MS et GS sont répartis en deux groupes, expérimental et témoin. Tous deux ont 8 séances d'écriture inventée, mais le groupe expérimental bénéficie d'un protocole d'intériorisation de l'auto-langage (se répéter l'énoncé à haute voix, puis à mi-voix et enfin dans sa tête) et d'aides à la relecture et à la réécriture. Les résultats montrent, outre une augmentation significative du nombre d'énoncés traités oralement dans le groupe expérimental, que la mobilisation de l'auto-langage aide l'enfant à analyser l'oral en le segmentant en petites unités sans risque de perdre le fil de l'énoncé. Il l'utilise à des fins de mémorisation immédiate (l'enfant rappelle verbalement l'information) et également pour contrôler son écriture (l'enfant relit ce qu'il a écrit). Ce type d'étude met en évidence l'origine sociale de l'apprentissage de l'écrit et ouvre la voie à une didactique procédurale (vs déclarative).

La remarque ayant été faite que certaines productions enfantines montrent l'emploi d'un nom de lettre pour coder une syllabe homophone (ex. vélo = VLO) - soit une « écriture littérale »une expérimentation didactique a été réalisée afin de vérifier si cette modalité peut varier selon le contexte didactique (Hannouz, 2008). La population est composée de deux classes de CP (expérimentale / témoin) auxquelles on demande pendant les tests (prétest, médian 1, médian 2, post-test) d'écrire des mots (ex. béton) contenant des syllabes se prononçant comme des noms de consonnes ([be], [de], [te], [ $\left.\nabla r_{\mathrm{r}}\right],\left[\bigotimes_{\mathrm{s}}\right]$ ). Lors de l'intervention didactique, les enfants du groupe expérimental écrivent, comme lors des tests, des mots contenant une syllabe homophone d'une consonne (ex. $\underline{\text { têt }}$ ) et des mots qui contiennent la même consonne mais associée à une voyelle autre que $[\mathrm{e}]$ ou $[\otimes]$; de ce fait, ces syllabes ne se prononcent pas comme un nom de lettre (ex. tasse). Une fois les mots écrits, l'expérimentateur interroge l'enfant sur le nombre de lettres employées pour écrire les deux syllabes. Pour les enfants qui utilisent une lettre pour coder la syllabe homophone (ex. $\underline{t e ̂} t u=\underline{T} T U$ ) et plusieurs lettres pour coder la syllabe non homophone (ex. tasse $=\underline{\mathrm{TAS}}$ ), la comparaison du nombre de lettres (ex. 1 lettre pour [te], 2 lettres pour [ta]) peut provoquer un conflit cognitif et amener l'enfant à modifier son codage.

L'analyse des résultats aux quatre tests dans les deux groupes montre une diminution plus rapide de l'emploi d'un nom de lettre pour le groupe expérimental. Les progrès des enfants de ce groupe semblent imputables à la mise en conflit du codage des syllabes homophone (ex. [te]) et non homophone (ex. [ta]). On note également davantage de procédures d'écriture plurielle dans le groupe expérimental que dans le groupe témoin (ex. les enfants codent la syllabe avec la consonne attendue et des lettres de remplissage : monté $=$ MTSO) ). Les différences observées entre les deux groupes permettent d'avancer que l'intervention didactique est source de progrès.

Une autre modalité observable peut être appelée "écriture lexicale». Certains enfants se basent en effet sur l'identité sonore entre la syllabe d'un mot et celle d'un mot-outil pour employer la graphie de ce

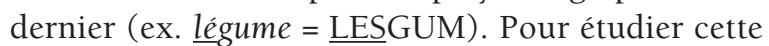
procédure d'écriture (Hannouz, 2008), on propose à des enfants de $\mathrm{CP}$ d'écrire des mots contenant des syllabes homophones de mots-outils (les, des, et, est) afin de mettre en évidence cette procédure, d'en comprendre les raisons et de tenter d'en diminuer expérimentalement l'emploi. Deux classes de CP 
(expérimentale / témoin) passent les tests (prétest, médian 1, médian 2 et post-test). Le groupe expérimental participe à neuf séances d'intervention didactique. Lors des tests les enfants écrivent des mots familiers (ex. légume), non familiers (ex. légal) ou des pseudo-mots (ex. Lémi). Pendant les séances d'intervention didactique, les enfants complètent une phrase avec un mot-outil et un mot contenant une syllabe homophone du mot-outil (ex. des coureurs sont sur la ligne de départ).

De manière générale, les résultats indiquent que l'emploi d'un mot-outil pour coder une syllabe homophone est une stratégie employée toute l'année par les enfants. Ils emploient de manière équivalente les mots-outil les, des, et ou est. L'analyse des arguments d'enfants qui recourent à ce traitement indique que la majorité se base sur l'identité sonore pour justifier l'emploi des mots-outil (ex Parce que j'entends pareil, alors j'écris pareil.). D'autres enfants emploient ces mots-outil, car selon eux les mots comportent un nombre minimum de lettres (ex. Parce qu'il est plus long. Et les mots c'est long; Quand on écrit, il faut mettre beaucoup de lettres). Outre la quantité de lettres, le principe de variabilité de l'écrit est pris en compte par des enfants pour justifier l'emploi des mots-outil (ex. On écrit comme on veut, [...] comme on pense.). D'autre part, la présence de la graphie $S$ dans les mots-outil les et des incitent certains enfants à les employer pour noter une quantité plurielle (ex. LESTU; [...] quand il y en a plus on met ce 'les').

La comparaison entre les deux groupes de l'emploi de l'écriture avec un mot-outil montre au post-test un emploi moindre pour les enfants du groupe expérimental que pour ceux du groupe témoin (5,1 \% / 13,8\%). Au-delà de l'analyse quantitative, l'analyse qualitative permet d'affiner les résultats des effets de l'intervention didactique. En effet, on observe que lors d'une même passation les enfants du groupe expérimental mettent en œuvre plusieurs traitements pour une syllabe donnée, tandis que ceux du groupe témoin n'explorent pas aussi facilement d'autres traitements. Même si les enfants du groupe expérimental ne décident d'abandonner l'écriture lexicale qu'en fin d'année, l'intervention didactique semble leur permettre de tester plus rapidement plusieurs hypothèses d'écriture. L'intervention facilite l'interaction de différentes connaissances graphiques.

\section{Conclusion}

Depuis plus de trente ans que l'écriture inventée est étudiée, les recherches effectuées dans les pays francophones amènent à la concevoir différemment aujourd'hui d'hier. Si la conception constructiviste sans laquelle elle ne présenterait aucun intérêt n'apparaît pas remise en cause, il n'en est pas de même du cas particulier que constitue la perspective piagétienne selon laquelle elle a été conçue. Des réplications effectuées en français, est ressortie en effet de manière constante l'impossibilité de retrouver la psychogenèse issue de travaux effectués principalement en espagnol. Les études d'ensemble conduites en des lieux et dates différents ont toutes conduit à des propositions autres que les propositions de départ. La difficulté à parvenir à des conclusions stables ressort de la diversité des évolutions proposées tant d'une équipe à l'autre qu'au sein d'une même équipe.

Méthodologiquement, il est alors apparu préférable de centrer la recherche sur des aspects particuliers, en procédant à des études ponctuelles, que de poursuivre la quête d'une psychogenèse universelle qui résistait à toutes les investigations. Les travaux se sont alors orientés sur les périodes énoncées. L'universalité de la première période et sa nécessité même ont été remises en cause. Pour la seconde période, les études permettent de retrouver des comportements interprétables en termes de quantité minimale, de variété intrafigurale et de variations quantitatives, mais la part relativement modeste de ces mécanismes dans les réponses montre la difficulté à faire de ceux-ci la pierre angulaire d'une période donnée : si certains enfants manifestent à l'évidence le recours à ces mécanismes, ce ne paraît pas être le cas de tous; les artefacts dus à la situation expérimentale incitent également à la prudence dans l'interprétation des réponses recueillies. Dans la troisième période, le rôle pivot conféré à la syllabe est apparu à son tour devoir être relativisé.

Ce que ce texte interroge, à l'issue des études présentées, c'est la généralisation à la langue française et au contexte francophone de l'évolution présentée par Ferreiro. Notre propos était limité à ce contexte, mais il serait intéressant de procéder à un semblable inventaire critique des travaux centrés sur ce même modèle dans des langues et des contextes différents, compte tenu notamment de l'affirmation 
souvent réitérée de sa validité pour des langues telles que l'italien, le portugais, le catalan, le français, l'anglais et le grec (Ferreiro, 2009). Au-delà de cette théorie particulière, pour une théorie générale de l'évolution de l'écriture chez l'enfant, il faudrait par ailleurs confronter ce modèle aux autres modèles évolutifs proposés dans le monde anglophone par des auteurs issus d'une autre tradition que la tradition piagétienne, tels que Ehri $(1987,1997)$, Frith (1985), Gentry (1982), Henderson (1981) et Treiman (1993, 1997, 1998, 2000). Enfin, au-delà de tel ou tel modèle particulier, de nombreux auteurs mettent en question l'idée que l'écriture se développe chez l'enfant selon un modèle par étapes (voir par exemple, Pacson et al, 1999, 2001; Rieben, 2003; Rieben, et al, 2005; Saada Robert, 2007). Notre propos, limité à un seul modèle et à un seul contexte linguistique, ne nous permet pas d'aller aussi loin.

Epistémologiquement, si la psychogenèse proposée par Ferreiro s'avère difficile à retrouver dans un contexte francophone et si les périodes qui la constituent posent problème, c'est semble-t-il du fait que des facteurs essentiels ne sont pas pris en compte par le modèle examiné. Prendre en considération le contexte, tant linguistique que didactique, apparaît alors comme nécessaire. Sur le plan linguistique, l'intérêt de comparaisons inter langues fait son chemin (Fijalkow, 2007). Sur le plan didactique, les comparaisons entre classes à didactique différente et les résultats qu'apportent les expérimentations didactiques convergent: quel que soit l'aspect de l'écrit examiné et quel que soit le mécanisme d'écriture examiné (dessiner / écrire; prénom / nom de famille; lettres homogènes / hétérogènes; segmentation de la phrase; autolangage; écriture littérale; écriture lexicale), la didactique a un effet sur les écrits produits. Voir dans quelle mesure l'écriture inventée dépend de la pensée de l'enfant (constructivisme), mais aussi du contexte linguistique et didactique (socioconstructivisme) apparaît dès lors comme un défi à relever par les recherches à venir. 


\section{NOTES}

1. Nous conserverons ici la formulation originelle d' « écriture inventée » («invented spelling »), bien que de nombreux auteurs aient tenté de trouver une formulation plus exacte - "écriture approchée ", "écriture enfantine ", " écriture provisoire ", " orthographe inventée », " orthographes inventées »...-. Aucune d'elles ne nous est apparue pleinement satisfaisante. Considérant alors que, de même que l'on dit " Pont neuf » sans s'interroger sur la pertinence actuelle du qualificatif «neuf », il est préférable de conserver une formulation qui, si imparfaite soit-elle, a cependant le mérite d'être parfaitement comprise de ses utilisateurs et de faciliter l'indexation des travaux qui y correspondent.

2. Pour un exemple, voir Rieben, Ntamakiliro, Gonthier et Fayol (2005) et Saada-Robert, M. (2007).

3. Il est nécessaire de distinguer la procédure alphabétique telle que définie ici par Ferreiro du principe alphabétique qui lui consiste à associer un graphème à un phonème.

4. Dans la présentation effectuée dans cet article, nous nous en tiendrons chaque fois que possible aux recherches effectuées dans un contexte francophone, pour des raisons de place et pour éviter les éventuels effets de contexte.

5. On emploiera systématiquement les abréviations GS (grande section), MS (moyenne section), PS (petite section), CP (cours préparatoire), CE1 (cours élémentaire lère année).

6. L'enfant est invité à relire ce qu'il a écrit en montrant du doigt où il l'a écrit. Cette procédure est destinée à mettre mieux en correspondance l'écrit de l'enfant, fragment par fragment, et ce qui lui a été demandé d'écrire.

7. Ils peuvent, par exemple, dire qu'un adulte n'écrirait pas comme eux.

8. Ces résultats, qui peuvent surprendre certains enseignants, sont sans doute à mettre en rapport avec la façon dont l'écriture du prénom est enseignée, généralement semble-t-il comme une unité insécable.

9. Cette interprétation, d'ordre psychanalytique, est une hypothèse qui demanderait une vérification faisant appel à d'autres techniques de recherche que celles utilisées par les travaux présentés dans ce texte.

10. Le cycle 2 regroupe 3 niveaux de classe : la GS de maternelle, le CP et le CE1.

11. Les Zones d'Education Prioritaire sont des zones géographiques rurales ou urbaines qui ont été créées en 1981 sur la base d'une politique de discrimination positive. Dotées de moyens spécifiques, elles mènent des actions éducatives en réseau des écoles et de collège, en partenariats avec les collectivités locales, les associations et institutions.

12. Les Réseaux d'Education Prioritaire ont succédé aux ZEP en 199, avec un contrat de réussite et 10 orientations prioritaires précises afin d'améliorer les objectifs pédagogiques et le pilotage.

13. Correspondances grapho-phonétiques

14. Au Québec, l'école maternelle dure un an (enfants de 5ans).

15. Le matériel d'entraînement ne renferme pas de mots dictés lors des tests.

16. La grille utilisée repose sur les grilles de Fijalkow et Fijalkow (1991) et Fijalkow et Liva (1993) ; compte tenu toutefois qu'il s'agit d'enfants de MS, elle n'utilise que les premiers échelons de celles-ci.

17. Ou «écriture orthographique»

18. Rieben et al (2005, p. 162) font un constat du même ordre dans une situation didactique montrant que les différences entre les groupes paraissent imputables à un tiers des élèves, ce qui renvoie à de fortes variations interindivuduelles.

19. Consonne-Voyelle (CV), Consonne-Consonne-Voyelle (CCV) et Consonne-Voyelle-Consonne (CVC)

20. Dans les derniers travaux de Ferreiro (Ferreiro, 2009 ; Molinari et Ferreiro, 2007), les analyses tiennent compte de la diversité des types de syllabes. 
L'ÉCRITURE INVENTÉE : EMPIRISME, CONSTRUCTIVISME, SOCIOCONSTRUCTIVISME

Jacques Fijalkow \& al

21. La diminution du nombre d'élèves entre les trois premières années s'explique par différents facteurs: déménagement, autorisation non remplie des parents ou refus de l'enseignante de faire passer aux enfants l'épreuve d'écriture.

22. Bien évidemment, cette expérience avait pour but de voir dans quelle mesure il est expérimentalement possible d'agir sur l'écriture à partir des caractères. Elle n'avait aucune visée de conseil pédagogique. 


\section{BIBLIOGRAPHIE}

Begin, C., Saint-Laurent, L., et Giasson J. (2005). La contribution des écritures provisoires dans la réussite en orthographe : étude longitudinale. Revue Canadienne de Linguistique Appliquée / CJAL. 8.2, 148-166.

Besse, J.-M. (1989). La production d'écrit chez le jeune enfant, avant l'école obligatoire. In J. Fijalkow (Eds.), Premier contact avec l'écriture et la lecture, (pp. 55-70). Rhodes: Helidoni,.

Besse, J.-M. (1990). L'enfant et la construction de la langue écrite. Revue Française de Pédagogie, 90, 17-22.

Besse, J.-M. (1992). L'écriture du prénom : apparence ou réalité d'une construction ? Les Dossiers de l'Éducation, $18,13-43$.

Besse, J.M. (2000). L'accès au principe phonographique : ce que montrent les écritures approchées. In G. Chauveau (Dir.), Comprendre l'enfant apprenti lecteur. Paris : Retz.

Bialystok, E., Shenfield T., et Codd, J. (2000). Languages, scripts, and the environment : Factors in developing concepts of print. Developmental Psychology, 36(1), 66-76.

Bialystok, E. (1997). Effects of bilingualism and biliteracy on children's emerging concepts of print. Developmental Psychology, 33, 429-440.

Bina, D., et Fijalkow, J. (soumis). Entre deux mots, comment choisir le moindre? Étude avec des enfants de cinq ans.

Catach, N. (1995). L'orthographe française, Paris : Nathan.

Cazes, V. (1996). Entrée dans l'écrit; étude de quelques stratégies. Thèse de doctorat, Université de Toulouse-2-Le Mirail, Toulouse, France.

Chomsky, C. (1971). Write first, read later. Childhood Education, 47, 296-299.

Creuzet, V., et Pasa, L. (2009). L'écriture inventée : que signifie écrire une lettre pour une syllabe?, Spirale, $44,31-48$.

David, J. (2002). Les débuts de la litéracie chez le jeune enfant. In G. Haas (Dir.), Apprendre, comprendre l'orthographe autrement, de la maternelle à l'université, Dijon : CRDP de Bourgogne.

David, J. (2003a). Linguistique génétique et acquisition de l'écriture. Faits de langue, 22, 1-11.

David, J. (2003b). La dimension orthographique dans les écrits des jeunes enfants. Les Dossiers des Sciences de l'Éducation, 9, 29-39.

De Gaulmyn, M.M. (1988, octobre). Langue écrite: la construction du système graphique par les enfants, Communication orale au congrès Connaissance et langage, Villeurbanne, France.

Dieguez, J., et Durand, A.-S. (2006). La perception de l'écrit chez l'enfant non-lecteur : notion de minimum/ maximum. Mémoire de master 1 non publié, Université de Toulouse-le Mirail, Toulouse, France.

Ehri, L. C. (1997). Learning to read and learning to spell are one and the same, almost. In C. Perfetti, L. Rieben, \& M. Fayol (Eds.), Learning to spell (pp. 237-269). Mahwah, NJ : Lawrence Erlbaum Associates, Inc.

Ehri, L. C., et Wilce, L. S. (1987). Does learning to spell help beginners learn to read words? Reading Research Quarterly, 22, 47-65.

Farre, C. (2000). La segmentation chez le jeune enfant. Thèse de doctorat, Université de Toulouse-2-Le Mirail, Toulouse, France.

Farre, C., et Fijalkow, E. (2009). La segmentation de la phrase par l'enfant. Intervention expérimentale. Spirale, 44, 63-82.

Ferreiro, E., et Teberosky, A. (1979). Los sistemas de escritura en el desarollo des nino, Siglo Veintuno Editores, Mexico.

Ferreiro, E., et Gomez-Palacio, M. (1988). Lire et écrire. Comment s'y apprennent-ils? Analyse des perturbations dans les processus d'apprentissage de la lecture et de l'écriture, Traduction française : Besse J.M., De Gaulmyn M., Ginet D., Lyon : CRDP. 


\section{L'ÉCRITURE INVENTÉE : EMPIRISME, CONSTRUCTIVISME, SOCIOCONSTRUCTIVISME}

Jacques Fijalkow \& al

Ferreiro, E. (2000). L'écriture avant la lettre. Hachette Éducation.

Ferreiro, E., et Teberosky, A. (1982). Literacy before schooling. Exeter, NH : Heinemann.

Ferreiro, E. (2009). La destabilizacion de las escrituras silabicas : alternancias y desorden con pertinencia. Lectura y Vida, 30(2), 6-13.

Ferreiro, E., Pontecorvo, C., et Zucchermaglio, C. (1987). Doppie o dopie? Eta Evolutiva, 27, 24-38.

Fijalkow, J. (2007). Invented Spelling in Varied contexts; a special guest issue of L1. L1-Educational Studies in Language and Literature, 7, issue 3.

Fijalkow, J., \& Fijalkow, E. (1991). L'écriture inventée au cycle des apprentissages. Dossiers de l'Éducation, 18, 125-167.

Fijalkow, J., \& Liva, A. (1993). Clarté cognitive et entrée dans l'écrit : construction d'un outil d'évaluation. In J.P. Jaffré, L. Sprenger-Charolles \& M. Fayol (Dir.), Lecture-écriture : acquisition; les actes de la Villette (pp. 203-229). Paris : Nathan.

Fijalkow, J., Fijalkow, E., Pasa, L., et Ragano, S. (2006). Entrer dans la culture écrite dans un contexte de langage entier. 3. L'écriture inventée. Caractères, 1, 26-48.

Frith, U. (1985). Beneath the surface of surface dyslexia. In K. E. Patterson, J. C. Marshall, \& M. Coltheart (Eds.), Surface dyslexia: Neuropsychological and cognitive studies of phonological reading (pp. 301-330). London : Routledge \& Kegan Paul.

Gentry, J. R. (1982). An analysis of developmental spelling in GNYS at WRK. The Reading Teacher, 36, 192-200.

Hannouz, D. (2008). Entrée dans l'écrit au CP. Études de quelques stratégies. Thèse de doctorat, Université de Toulouse-2-Le Mirail, Toulouse, France.

Henderson, E. H. (1981). Learning to read and spell : A child's knowledge of words. Dekalb : Northern Illinois University Press.

Hjelmslev, L. (1971). Essais linguistiques, Paris : Ed. Minuit.

Jaffré, J.P. (1992). Le traitement élémentaire de l'orthographe : les procédures graphiques. Langue Française, 95, 27-48.

Jaffré, J.P., Bousquet, S., et Massonnet, J. (1999). Retour sur les orthographes inventées. Les Dossiers des Sciences de l'Éducation, 1, 39-52.

Lautrey, J., Rémi-Giraud, S., Sander, E., et Tiberghien, A. (2009). Les connaissances naïves, Paris : A. Colin.

Luis, M.H. (1993). Contribution à l'étude de la psychogenèse de l'écrit. Une expérimentation en banlieue lyonnaise. Thèse de doctorat, Université Lumière Lyon 2 , Lyon, France.

Masse, J., \& Tenes, V. (2006). La variété intrafigurale. Mémoire de master 1, Université de Toulouse-le Mirail non publié, Toulouse, France.

Molinari, C., et Ferreiro, E. (2007). Identidades y diferencias en las primeras etapas del proceso de alfabetización. Lecture y Vida, 4, 18-30.

Montesinos-Gelet, I., et Morin, M.-F. (2001). S’approcher de la norme orthographique en lère année du primaire : qu'en est-il de la pluralité des conceptions linguistiques? Archives de Psychologie, 69 (270-271), 159-176.

Morin, M.-F. (2004a). Comprendre et prévenir les difficultés en écriture chez le jeune enfant en examinant les orthographes approchées et les commentaires métagraphiques. In J.-C. Kalubi, \& G. Debeurme (Dir.), Identité professionnelle et interventions scolaires. Contextes de formation de futurs enseignants. (pp. 145-173), Sherbrooke : CRP.

Morin, M.-F. (2004b). Les niveaux d'explicitation des connaissances sur la morphographie du nombre au début du primaire. In C. Brissaud et C. Totereau, Acquisition et enseignement de l'orthographe, Revue de Linguistique et de Didactique des Langues, 30, 55-72. 
Morin, M.-F. (2004c). Un portrait évolutif des habiletés orthographiques d'écoliers québécois entre 6 et 7 ans. In C. Barré-de Miniac, C. Brissaud et M. Rispail (Eds.), La littéracie : conceptions théoriques et pratiques d'enseignement de la lecture-écriture (pp. 193-207). Paris : L'Harmattan.

Morin, M.-F. (2005). Declared knowledge of beginning writers. L1 - Educational Studies in Language and Literature, 5, 385-401.

Morin, M.F., et Montesinos-Gelet, I. (2005). Les habiletés phonogrammiques en écriture à la maternelle: comparaison de deux contextes francophones différents, France-Québec. Revue Canadienne de l'Éducation, $28(3), 1-23$.

Morin, M-F., et Montesinos-Gelet, I. (2004). La diversité des procédures utilisées par le jeune enfant scripteur en début d'apprentissage de l'écrit. Scientia Paedagogica Experimentalis, XLI, 2, 301-325.

Noyer, M. (2005). Écrire avant de savoir écrire. Enfance 2005/1, 57, pp. 11-23.

Noyer, M., et Baldy, R. (2005). Influence de la complexité des formes imagées et langagières d'un référent et rôle du prénom dans l'acquisition de l'écriture entre trois et six an. Archives de Psychologie, 71, no278-79, $199-215$.

Noyer-Martin, M., et Baldy, R. (2008). L'acquisition de l'écriture chez les enfants de 3 à 8 ans : approche évolutive transverse. Bulletin de Psychologie, 61 (5), 497, 449-459.

Pacton, S., Fayol, M. et Perruchet, P. (1999).L’apprentissage de l'orthographe lexicale : le cas des régularités. Langue Française, 124, 24-39.

Pacton, S., Fayol, M. et Perruchet, P., et Cleeremans, A. (2001). Implicit learning out of the lab : The case of orthographic regularities. Journal of Experimental Psychology. General, 130(3), 401-423.

Pasa, L. (2002a). Apprentissage de l'écriture dans deux CP contrastés. Voies livres, V99, janvier 2002.

Pasa, L. (2002b). Entrer dans la culture écrite : Rôle des contextes pédagogiques et didactiques. Thèse de doctorat, Université de Toulouse-2-Le Mirail, Toulouse, France.

Pasa, L., et Morin, M.-F. (2007). Beginning spelling and literacy approaches : A comparative study between French and Quebecois first-grade classes. L1- Educational Studies in Language and Literature, 3 (Special issue on Invented Spelling).

Pasa, L., Creuzet, V., et Fijalkow, J. (2006). Écriture inventée : pluralité des traitements et variabilité selon la structure syllabique. Éducation et Francophonie, XXXIV(2), p. 149-167..

Pomel, J. (2008). Conscience du langage et auto-langage chez l'enfant apprenti-scripteur, Thèse de doctorat, Université de Toulouse-le Mirail, Toulouse, France.

Read, C. (1975). Children's Categorizations of Speech Sounds in English, Urbana, 111, National Council of Teachers of English.

Read, C. (1981). Writing is not the inverse of reading for young children. In C. H. Fredericksen \& J.F. Dominic (Eds.), Writing: The nature, development, and teaching of written communication (pp. 105-115). Hillsdale.

Rieben, L. (2003). Écritures inventées et apprentissage de la lecture et de l'orthographe. Faits de langue, déc. $22,27-36$.

Rieben, L., Ntamakiliro, L., Gonthier, B., et Fayol, M. (2005). Effects of Various Early Writing Practices on Reading and Spelling. Scientific Studies of Reading, 9(2), 145-166.

Saada-Robert, M. (2007). Produire des écrits pour apprendre à lire. In ONL. Écrire des textes, l'apprentissage et le plaisir.

Sarris, D. (1996). L'écriture inventée : psychogenèse ou sociogenèse? Thèse de doctorat, Université de Toulouse-le Mirail, Toulouse, France.

Treiman, R., et Cassar, M. (1997). The beginning of orthographic knowledge : Children's knowledge of double letters in words. Journal of Educational Psychology, 89, 613-644.

Treiman, R. (1993). Beginning to spell : A study of first-grade children. NewYork : Oxford University Press.

Vernon, S.A., et Ferreiro, E. (1999). Writing development : a neglected variable in the consideration of phonological awareness. Harvard Educational Review, 69, 4, 395-415. 